\title{
Boosting Lithium-Sulfur Battery Performance by Integrating Redox-Active Covalent Organic Framework in the Separator
}

Qing Xu, ${ }^{\mathrm{a}}+$ Kailong Zhang, ${ }^{\mathrm{b}}+$ Jing Qian,,${ }^{\mathrm{a}}$ Yu Guo, ${ }^{\mathrm{a}}$ Xiaokai Song, ${ }^{\mathrm{b}}$ Honglin Pan, ${ }^{\mathrm{b}}$ Di Wang,,${ }^{\mathrm{b}}$ and Xiaopeng $\mathrm{Li}^{\mathrm{a}}{ }^{\text {** }}$

a. CAS Key Laboratory of Low-Carbon Conversion Science and Engineering, Shanghai Advanced Research Institute (SARI), Chinese Academy of Sciences (CAS), Shanghai 201210, China

b. Resource Environment and Clean Energy Laboratory, School of Chemistry and Environment Engineering, Jiangsu University of Technology, Changzhou 213001, China

E-mail: lixp@sari.ac.cn

Table of Contents

Section 1. Methods .2

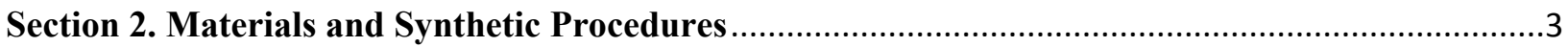

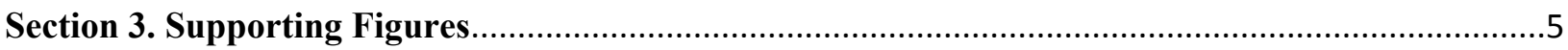

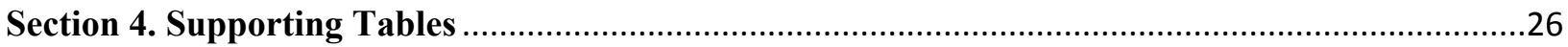

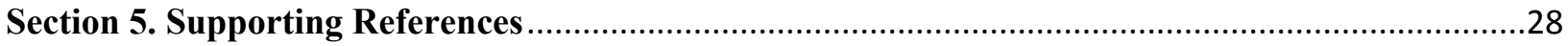




\section{Section 1. Methods}

Characterizations. Powder X-ray diffraction (PXRD) data were recorded on a Rigaku model RINT Ultima III diffractometer by depositing powder on glass substrate, from $2 \theta=1.5^{\circ}$ up to $60^{\circ}$ with $0.02^{\circ}$ increment. Nitrogen sorption isotherms were measured at $77 \mathrm{~K}$ with a Micromeritics Instrument Corporation model 3Flex surface characterization analyzer. The Brunauer-EmmettTeller (BET) method was utilized to calculate the specific surface areas. By using the non-local density functional theory (NLDFT) model, the pore volume was derived from the sorption curve. TGA measurements were performed on a Mettler-Toledo model TGA/SDTA851e under $\mathrm{N}_{2}$, by heating to $800^{\circ} \mathrm{C}$ at a rate of $10^{\circ} \mathrm{C} \mathrm{min}^{-1}$. FE-SEM images were obtained on a FEI Sirion-200 or Hitachi high technologies (SU-6600) field-emission scanning electron microscope at an electric voltage of $5 \mathrm{KV}$. X-ray photoelectron spectroscopy (XPS) experiments were carried out on an AXIS Ultra DLD system from Kratos with $\mathrm{Al} \mathrm{K} \alpha$ radiation as X-ray source for radiation. EDX and elemental mapping were acquired using a HITCHI Miniscope TM3030. 


\section{Section 2. Materials and Synthetic Procedures}

Materials. Dimethylacetamide (DMAC), 1, 2-Dichlorobenzene (ODCB) Acetic acid, tetrahydrofuran (THF), methanol (MeOH) and Benzidine dihydrochloride (BPD) were obtained from Aladin. 2, 4, 6-trihydroxybenzene-1, 3, 5-tricarbaldehyde (TP) [2, 2'-bipyridine]-5, 5'diamine (BPY), were purchased from Jilin Province Extension Technology Co., Ltd. Lithium bis (trifluoromethane sulfonimide) (LiTFSI), $\mathrm{LiNO}_{3}$ 1, 3-dioxolane (DOL), 1, 2-dimethoxyethane (DME), polyvinylidene fluoride (PVDF) binder and N-methylpyrrolidinone (NMP) were purchased from Aldrich.

Synthesis of TP-BPY-COF. A mixture of DMAC/oDCB (0.9 mL/0.3 mL), TP (12.6 mg), BPY $(16.7 \mathrm{mg})$ and aqueous acetic acid solution $(6 \mathrm{M}, 0.12 \mathrm{~mL})$ was ultrasonicated for several minutes, and then degassed in a Pyrex tube $(10 \mathrm{~mL})$ by three freeze-pump-thaw cycles. The tube was sealed off and heated at $120^{\circ} \mathrm{C}$ for 3 days. The precipitate was collected by centrifugation, washed with THF, HAc ( $3 \mathrm{M})$ aqueous solution and $\mathrm{MeOH}$ for several times, and dried at $120^{\circ} \mathrm{C}$ under vacuum overnight to obtain TP-BPY-COF in $82 \%$ yield.

Synthesis of TP-BPD-COF. A mixture of DMAC/oDCB (0.9 mL/0.3 mL), TP (12.6 mg), BPD $(23.1 \mathrm{mg})$ and aqueous acetic acid solution $(6 \mathrm{M}, 0.12 \mathrm{~mL})$ was ultrasonicated for several minutes, and then degassed in a Pyrex tube $(10 \mathrm{~mL})$ by three freeze-pump-thaw cycles. The tube was sealed off and heated at $120^{\circ} \mathrm{C}$ for 3 days. The precipitate was collected by centrifugation, washed with THF, $\mathrm{HAc}(3 \mathrm{M})$ aqueous solution and $\mathrm{MeOH}$ for several times, and dried at $120^{\circ} \mathrm{C}$ under vacuum overnight to obtain TP-BPD-COF in $78 \%$ yield.

Prepare complex of $\mathbf{S}$ and carbon black. Sulfur-loaded super $\mathrm{P}$ was prepared via calcination of the ball-milled mixture of sulfur and super $\mathrm{P}$ at $155{ }^{\circ} \mathrm{C}$ for $4 \mathrm{~h}$ in $\mathrm{N}_{2}$ atmosphere. The mass of sulfur loading is about $1-1.5 \mathrm{mg} \mathrm{cm}^{-2}$

Prepare COF-based separator. The slurry ( $60 \mathrm{wt} \%$ of TP-BPY-COF or TP-BPD-COF, $30 \mathrm{wt} \%$ of Super $\mathrm{P}$ and $10 \mathrm{wt} \%$ of PVDF in NMP), was coated onto the Celgard membrane, and dried at $55^{\circ} \mathrm{C}$ under vacuum overnight to obtain TP-BPY-COF based separator and TP-BPD-COF based separator.

Prepare polysulfides and organic electrolyte. The $\mathrm{Li}_{2} \mathrm{~S}_{6}$ solution was prepared by adding sublimed $\mathrm{S}$ and $\mathrm{Li}_{2} \mathrm{~S}$ in a molar ration of 5:1 by stoichiometrically into anhydrous tetrahydrofuran (THF) at the same time in argon-filled glovebox. Then aging for $10 \mathrm{~h}$ and homogeneous solution was obtained. The electrolyte consisted of LiTFSI $\left(1 \mathrm{~mol} \mathrm{~L}^{-1}\right)$ and $\mathrm{LiNO}_{3}(1 \mathrm{wt} . \%)$ in a DOL-DME solution (1:1 ratio in volume).

Lithium-sulfur batteries fabrication. CR 2016-type coin cells were assembled in an argon-filled glovebox by using Li foil as the counter electrode, the complex of super $\mathrm{P}$ and $\mathrm{S}$ as work electode, 
and TP-BPY-COF-based membrane, TP-BPD-COF-based membrane, or Celgard as separators. All electrochemical measurements were performed at $25^{\circ} \mathrm{C}$.

Lithium ion diffusion coefficient can be calculated as follow:

\section{Calculation of lithium ion diffusion coefficient:}

$\mathrm{CV}$ measurements were scanned between 1.7 and $2.8 \mathrm{~V}$ at $0.1 \mathrm{mV} \mathrm{s}^{-1}$ and the lithium ion diffusion coefficient was calculated by the Randles-Sevick equation:

$$
\mathrm{I}_{\text {peak }}=2.69 \times 10^{5} \times n^{1.5} \times A \times D_{L i^{+}}^{0.5} \times C_{L i^{+}} \times V^{0.5}
$$

Where $I_{\text {peak }}$ is the peak current (A), $\mathrm{n}$ is the number of electrons (For LSBs is 2), A is area of the cathode $\left(\mathrm{cm}^{-2}\right), C_{\mathrm{Li}}$ is the change of Li ions in the electrolyte $\left(\mathrm{mol} \mathrm{L}^{-1}\right)$, and $v$ is the $\mathrm{CV}$ scanning rate $\left(\mathrm{V} \mathrm{s}^{-1}\right) \cdot{ }^{\mathrm{S} 3}, \mathrm{~S} 4$ 


\section{Section 3. Supporting Figures}
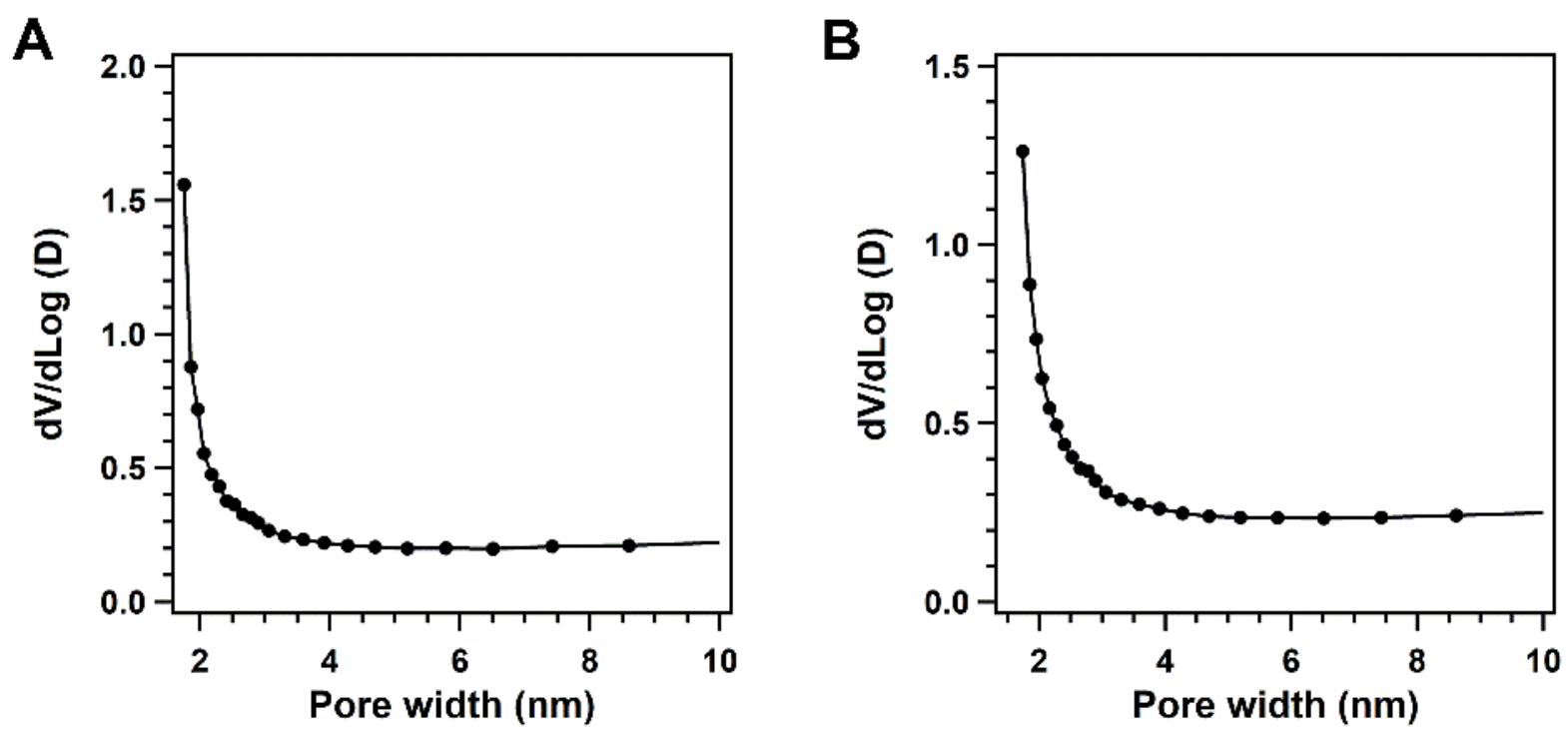

Figure S1. Pore size distribution profiles of TP-BPY-COF (A) and TP-BPD-COF (B). 


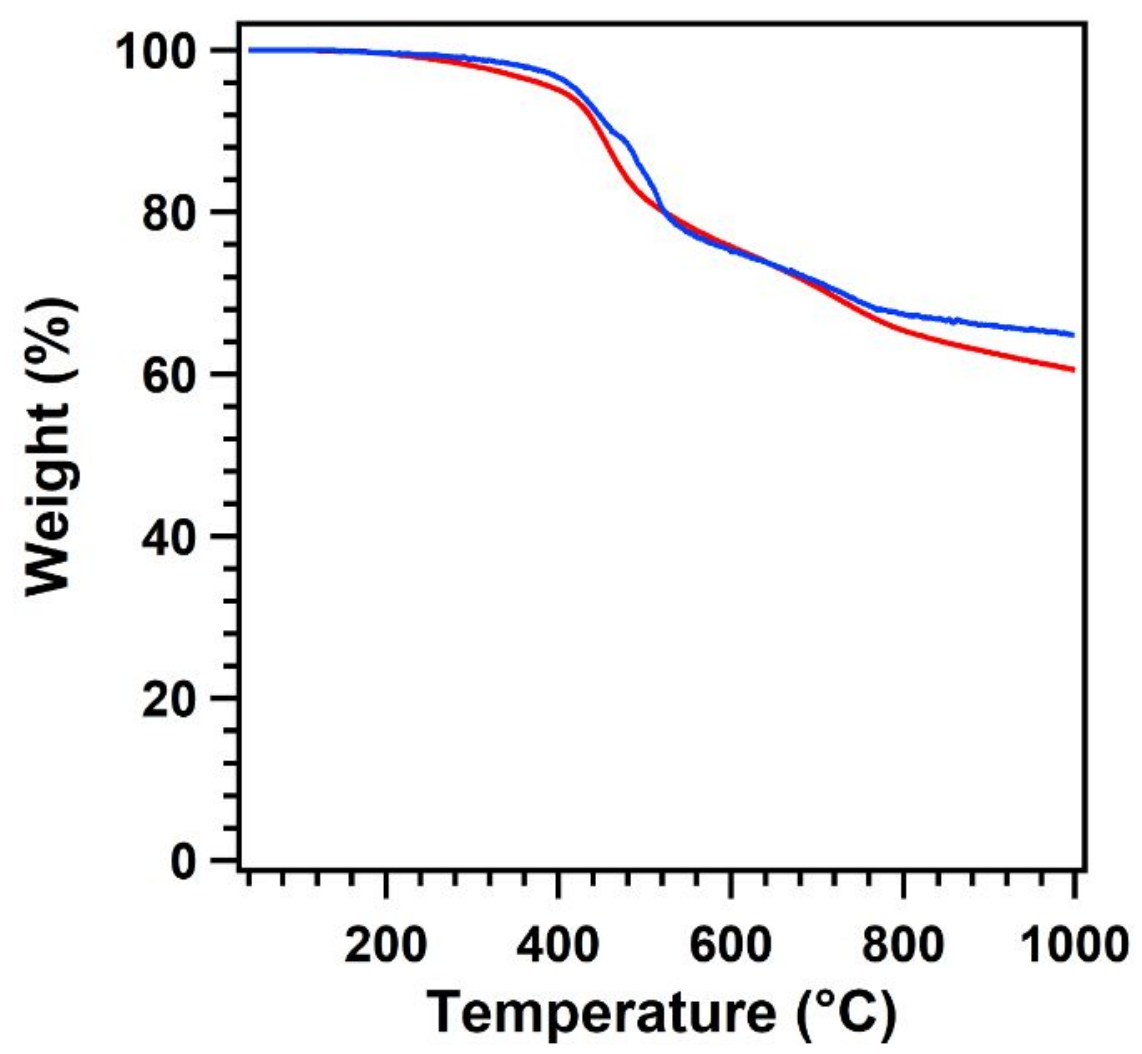

Figure S2. TGA curves of TP-BPY-COF (red) and TP-BPD-COF (blue) under $\mathrm{N}_{2}$. 

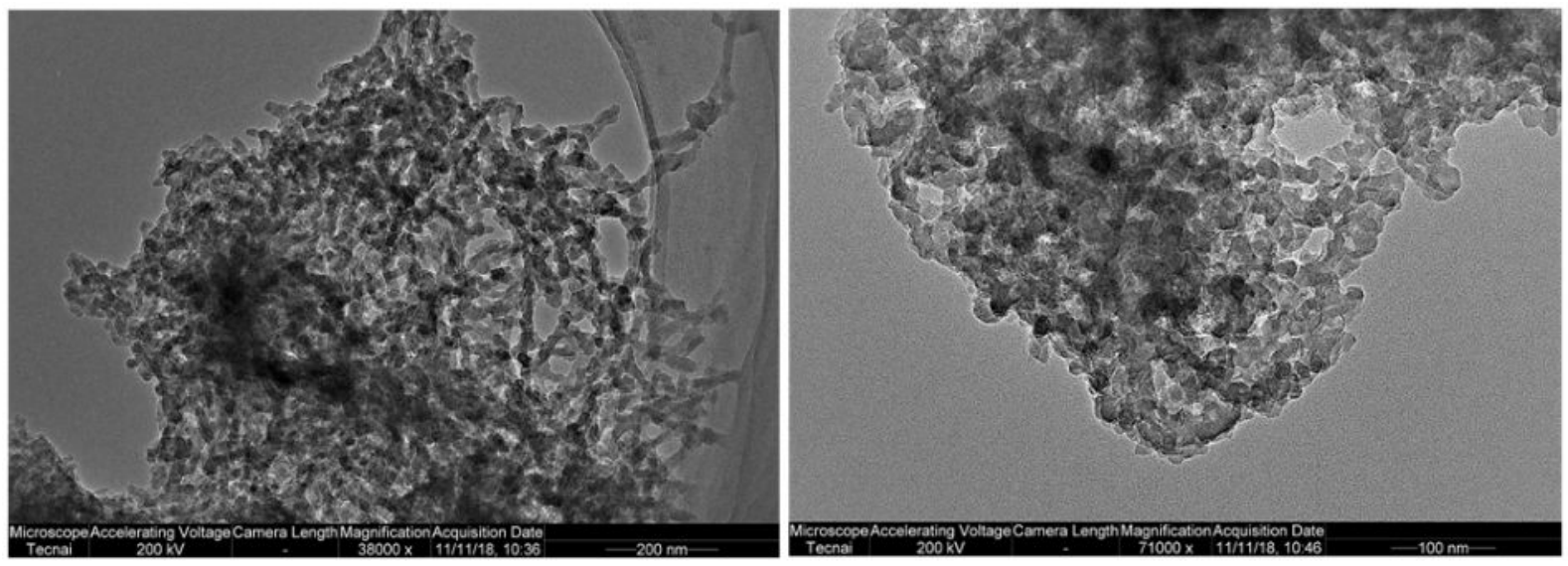

Figure S3. TEM images of TP-BPY-COF. 

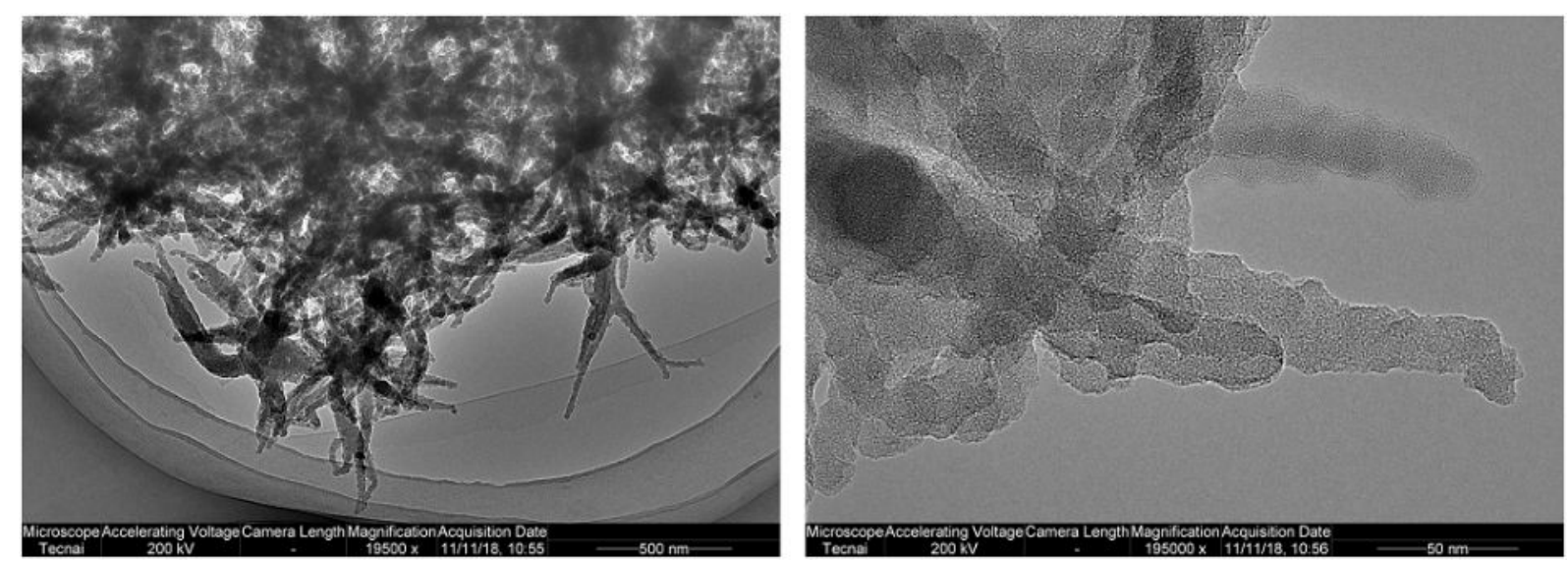

Figure S4. TEM images of TP-BPD-COF. 
Electron Image 1

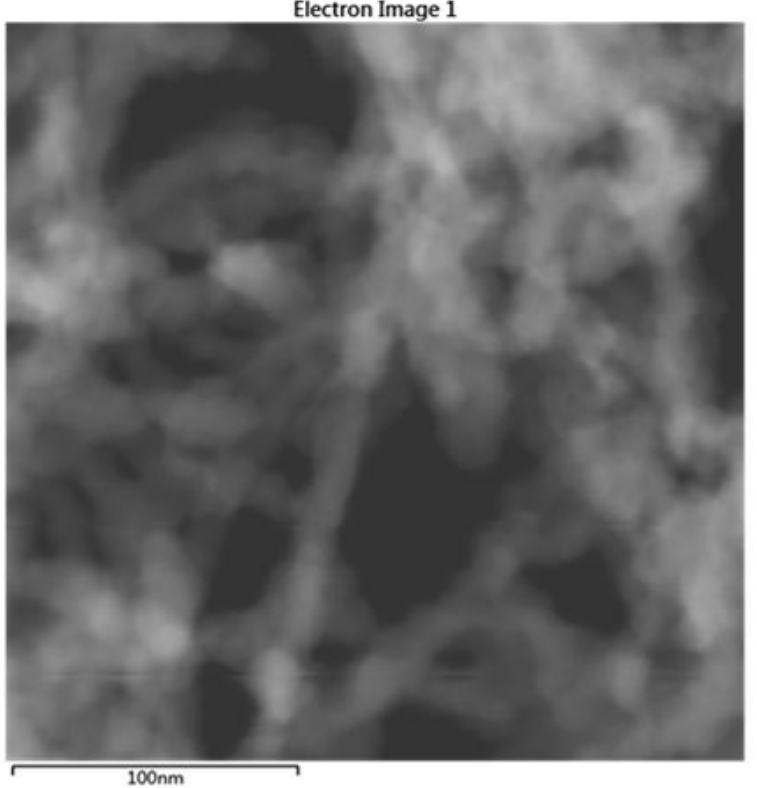

$100 \mathrm{~nm}$

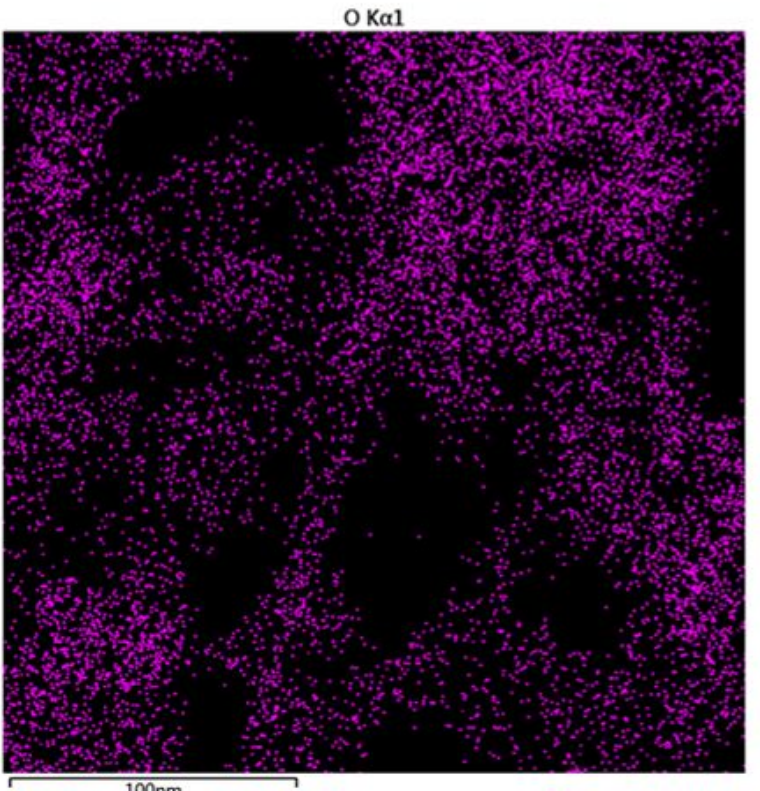

$100 \mathrm{~nm}$

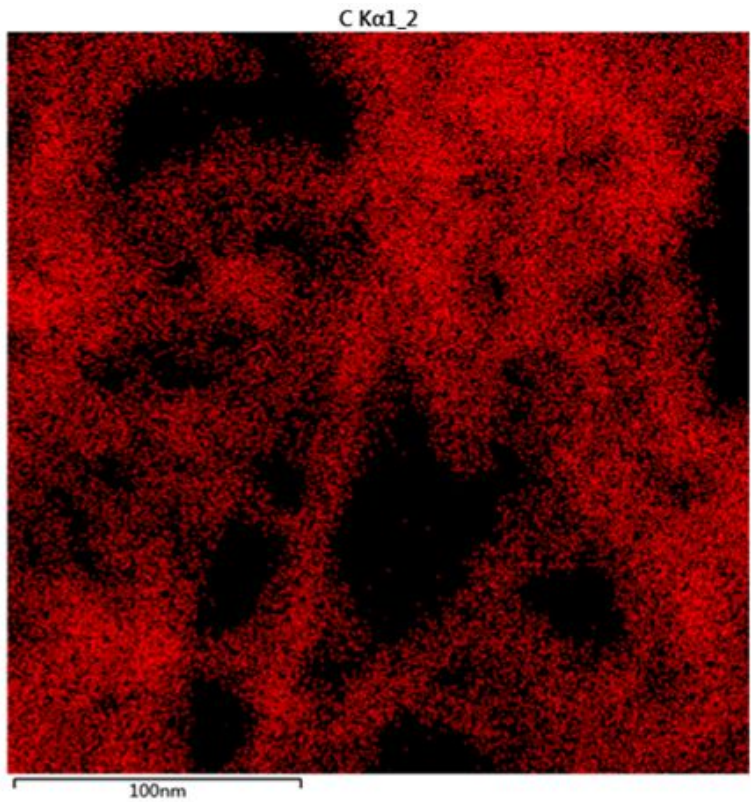

N Kal 2

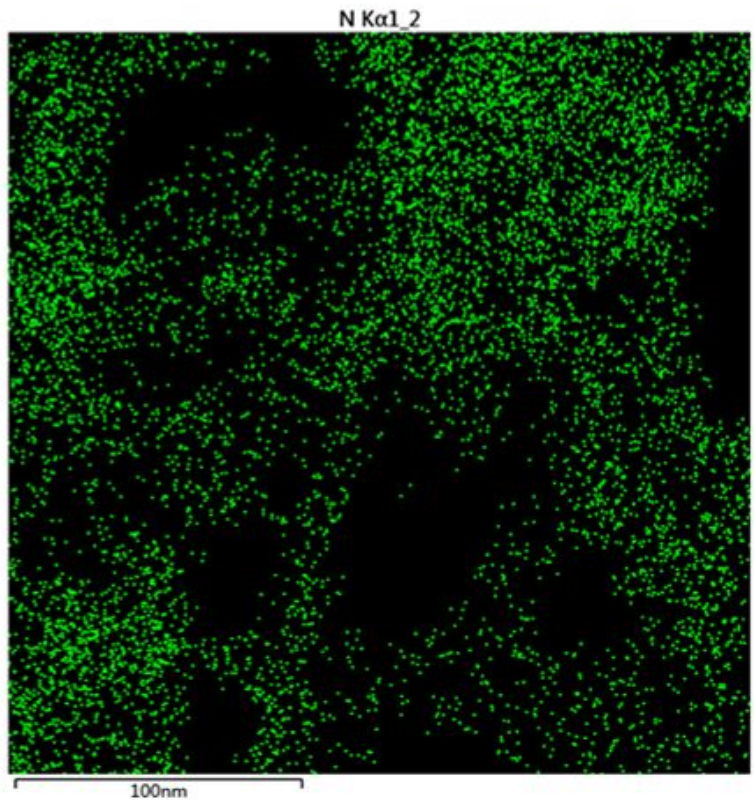

Figure S5. TEM-mapping images of TP-BPY-COF. 

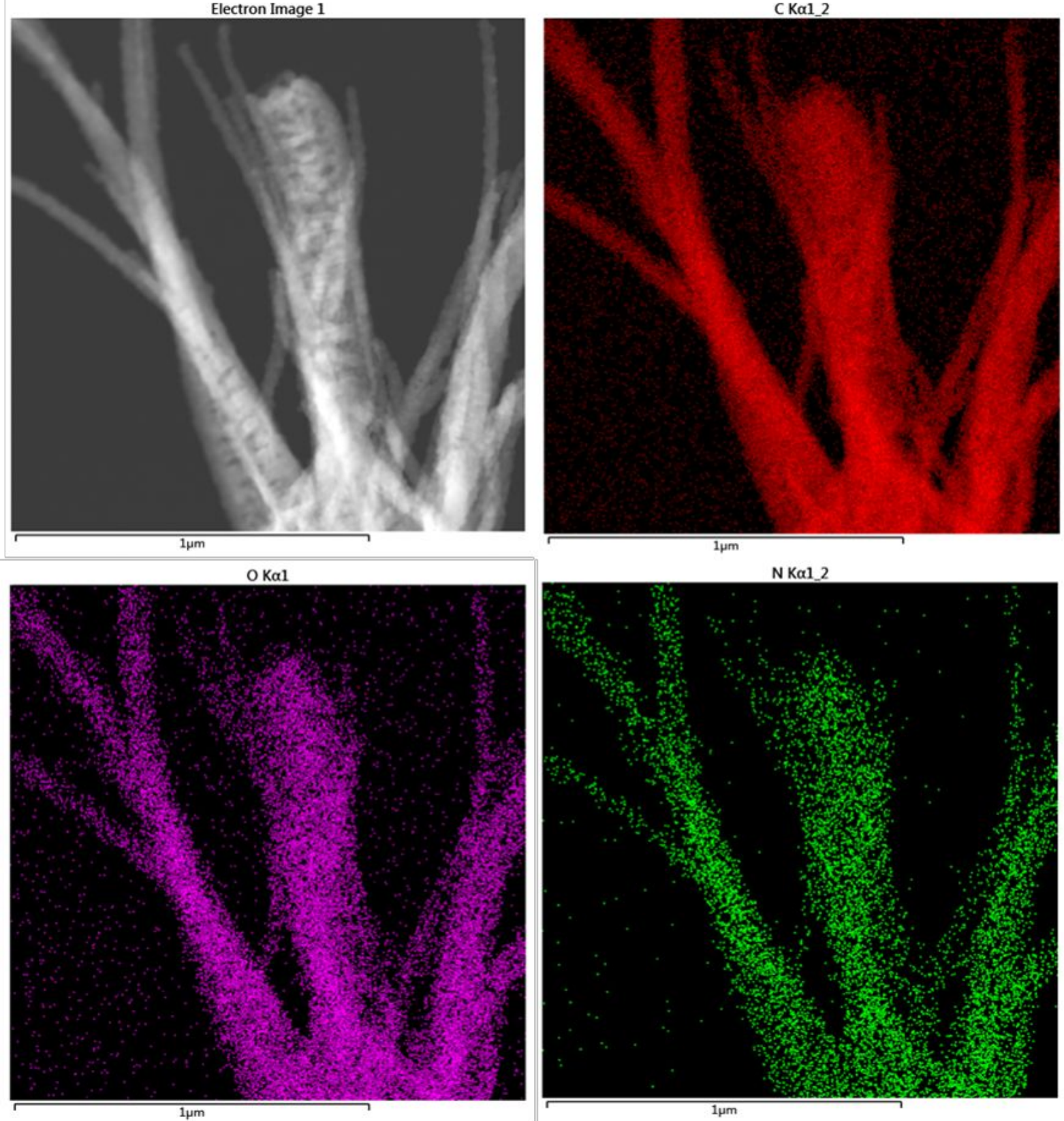

Figure S6. TEM-mapping images of TP-BPD-COF. 


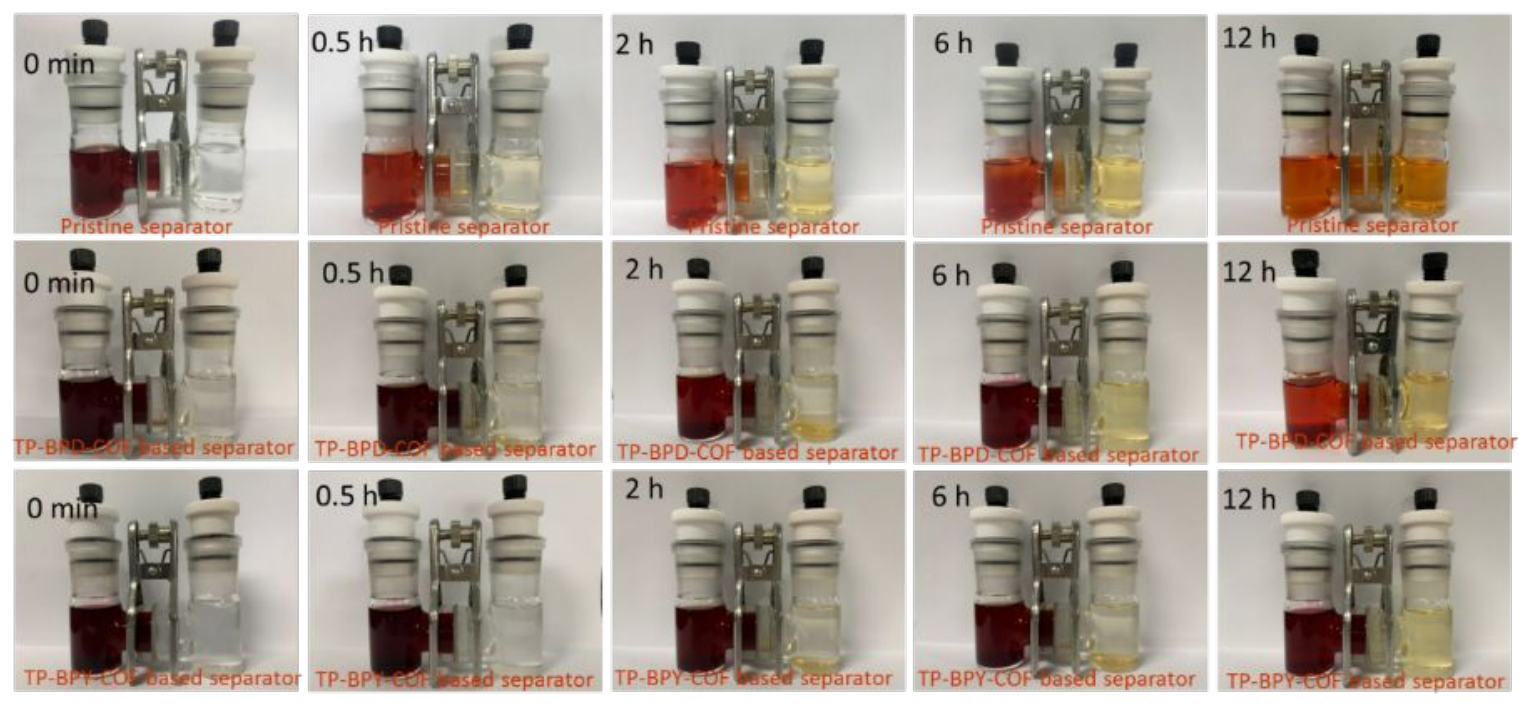

Figure S7. Optical images for the generation and diffusion of PS in H-type cells with different separator. 


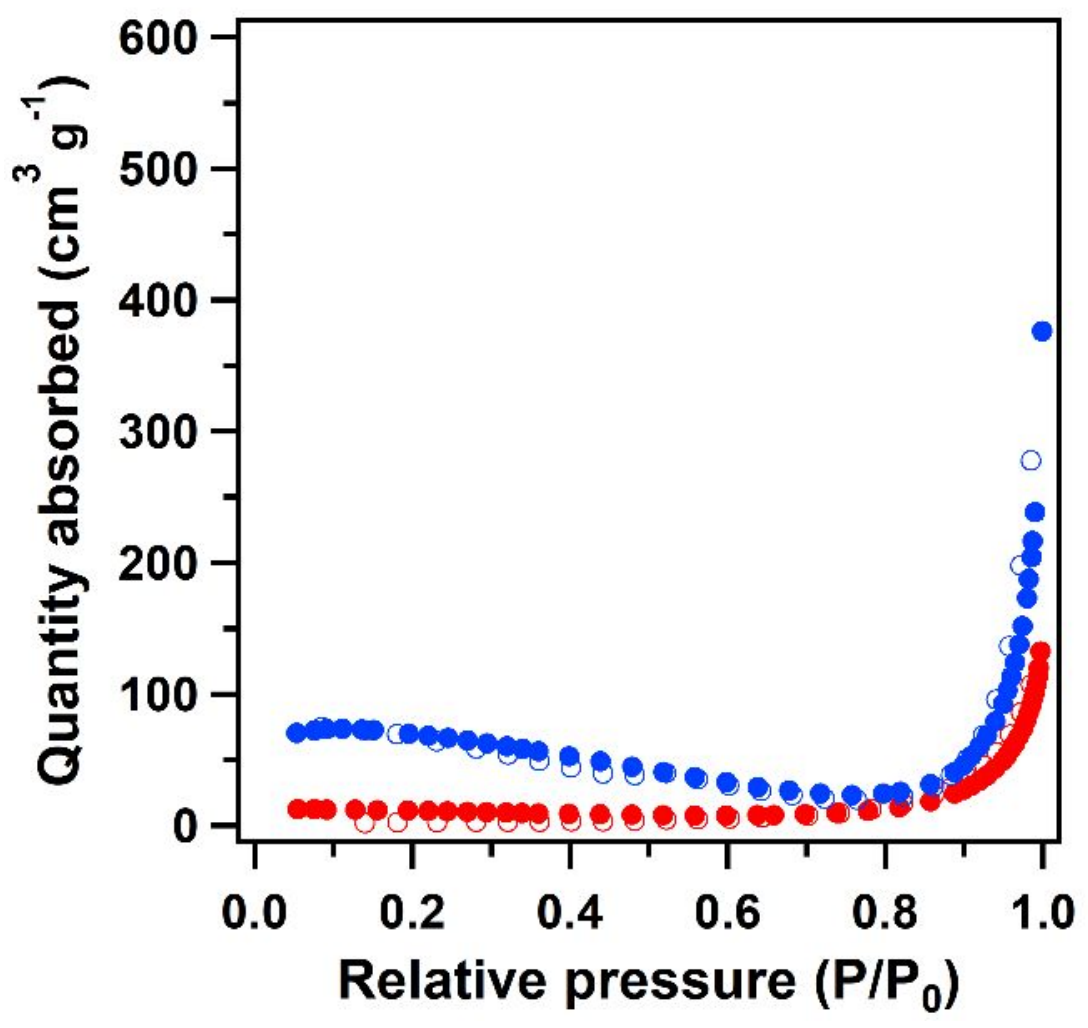

Figure S8. Nitrogen isothermal profiles of $\mathrm{Li}_{2} \mathrm{~S}_{6} @ \mathrm{TP}-\mathrm{BPY}-\mathrm{COF}$ (red) and $\mathrm{Li}_{2} \mathrm{~S}_{6} @ \mathrm{TP}-\mathrm{BPD}-\mathrm{COF}$ (blue). 


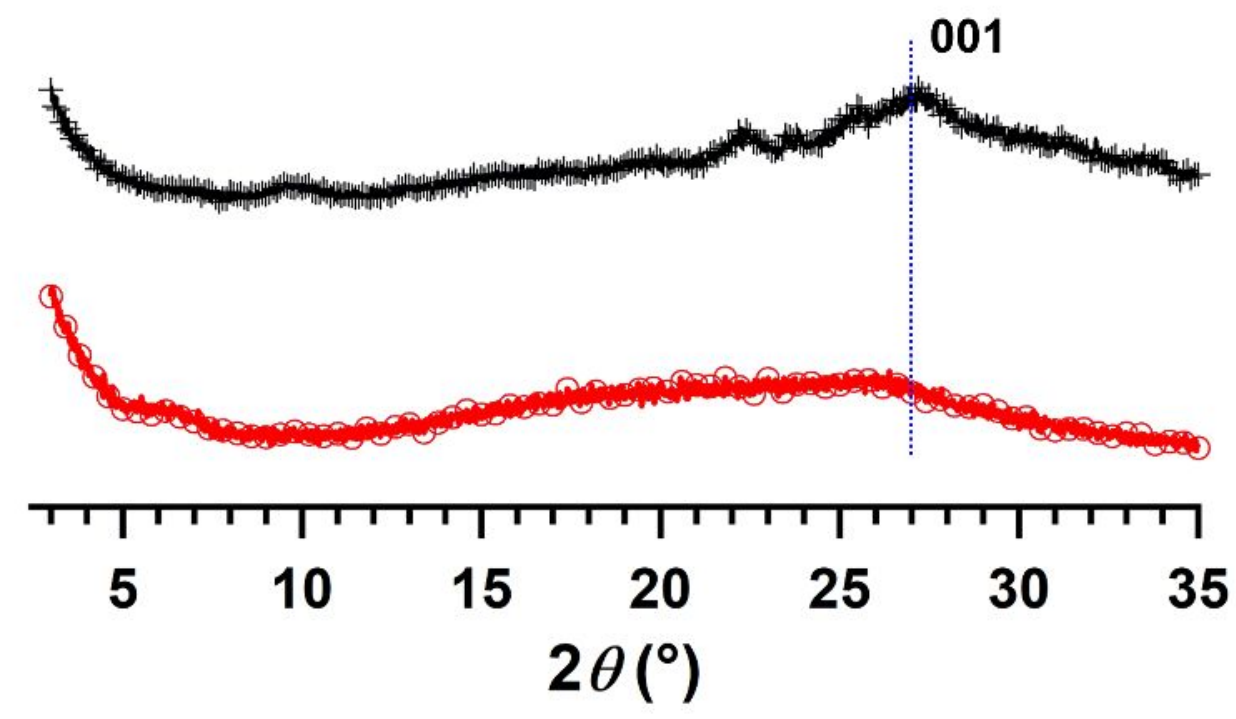

Figure S9. PXRD profiles of $\mathrm{Li}_{2} \mathrm{~S}_{6} @$ TP-BPY-COF (red) and Li $\mathrm{S}_{6} @$ TP-BPD-COF (black). 
A

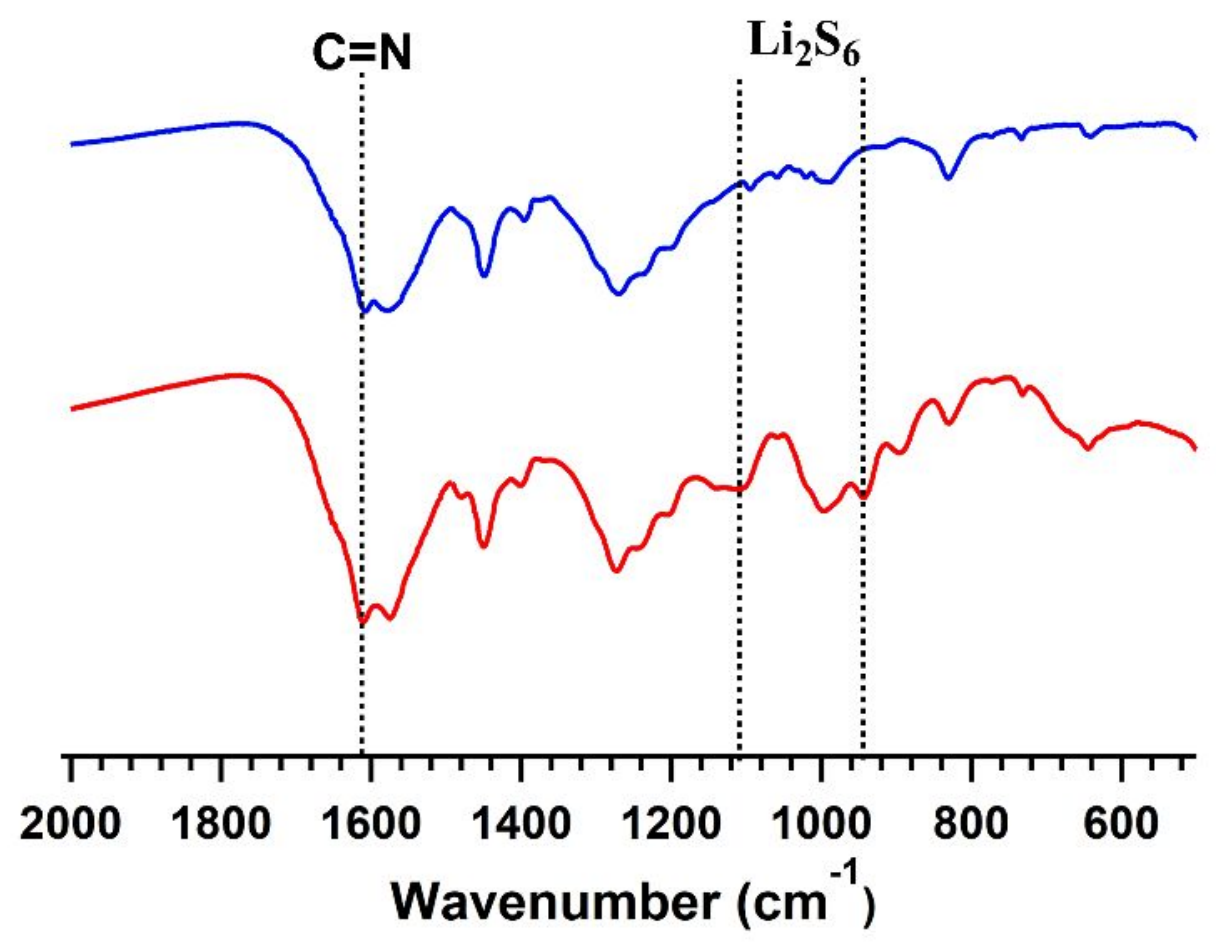

B

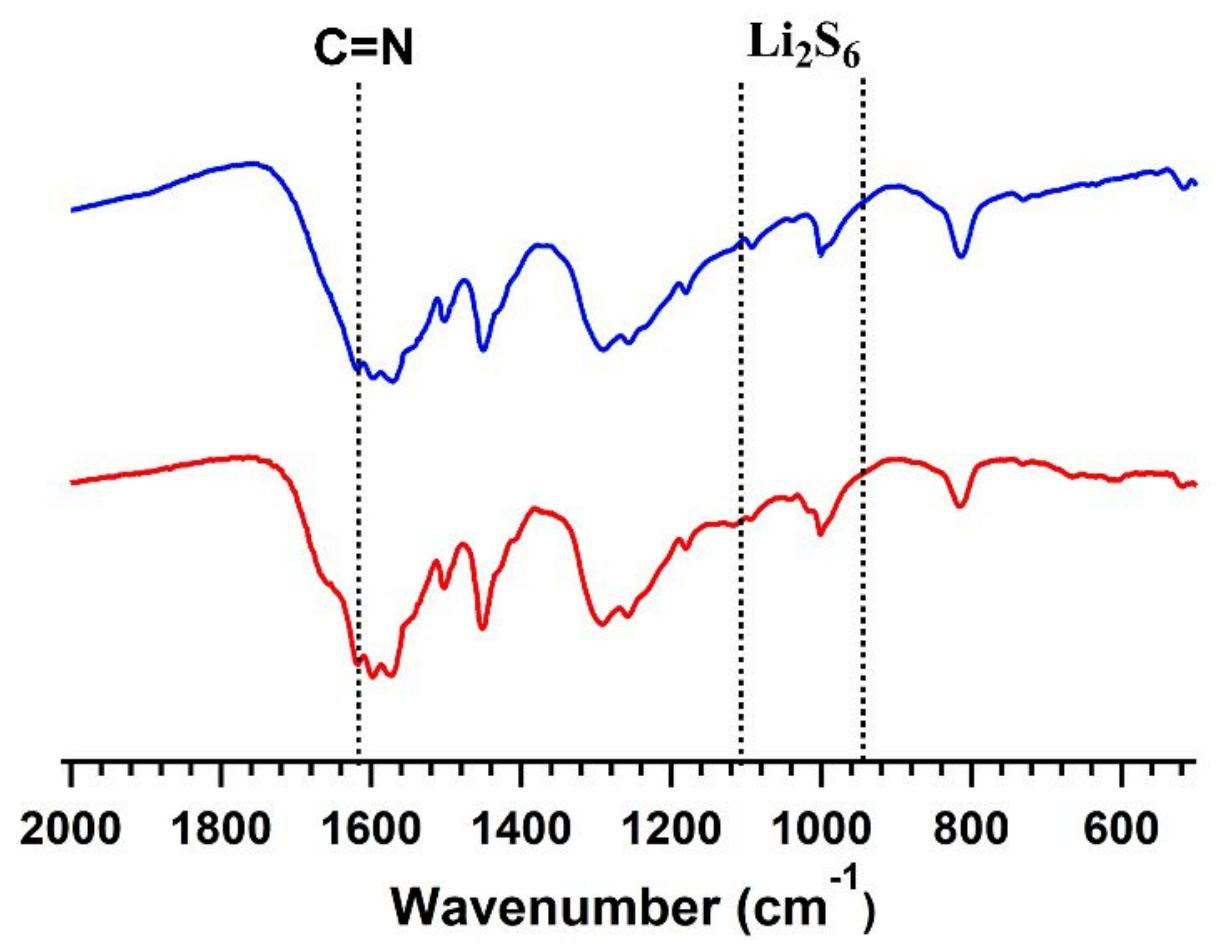

Figure S10. FT IR spectra of (A) TP-BPY-COF (blue) and $\mathrm{Li}_{2} \mathrm{~S}_{6} @$ TP-BPY-COF (red). FT IR spectra of (B) TP-BPD-COF (blue) and $\mathrm{Li}_{2} \mathrm{~S}_{6} @$ TP-BPD-COF (red). 


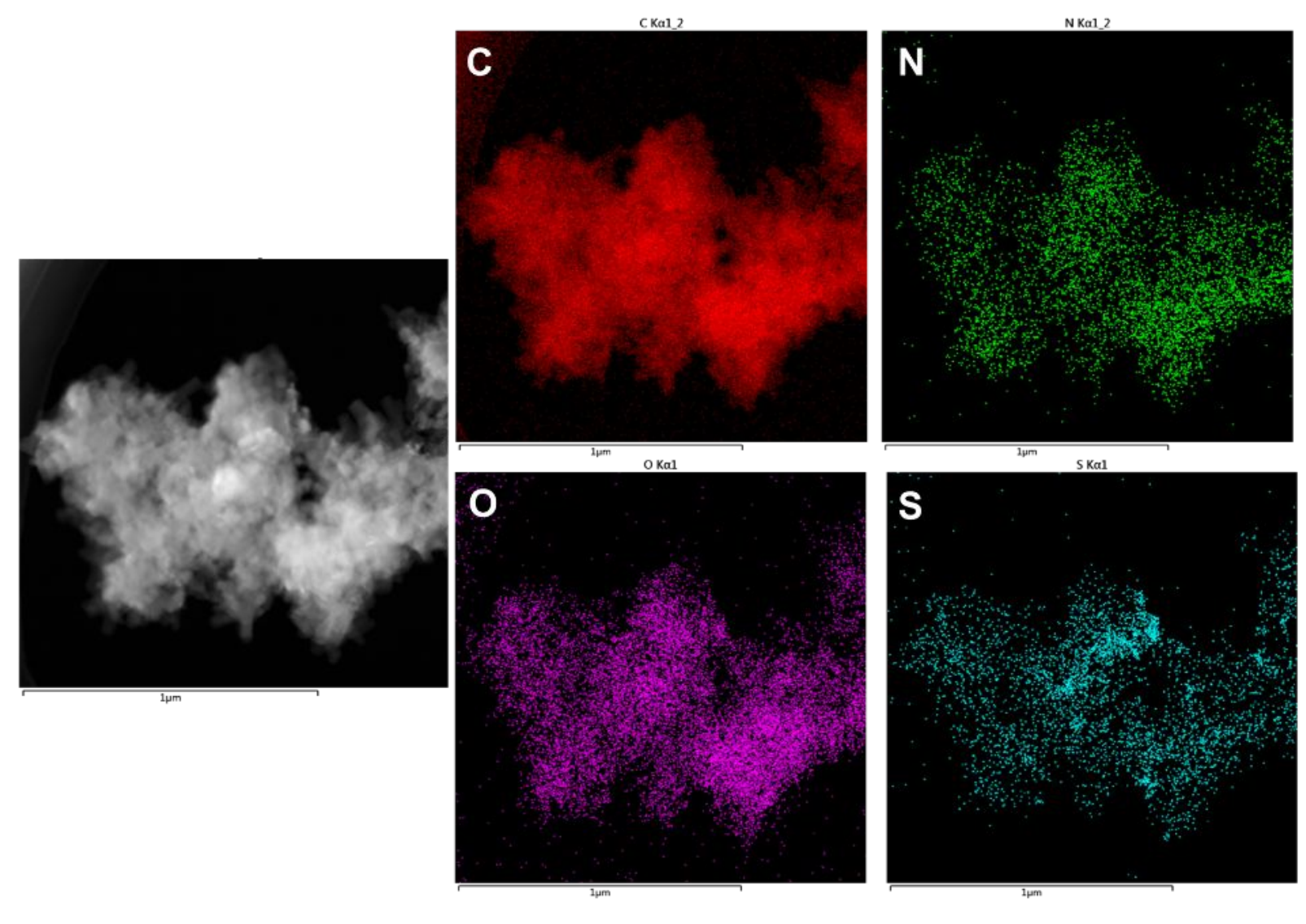

Figure S11. TEM-mapping of $\mathrm{Li}_{2} \mathrm{~S}_{6} @ \mathrm{TP}-\mathrm{BPD}-\mathrm{COF}$. 

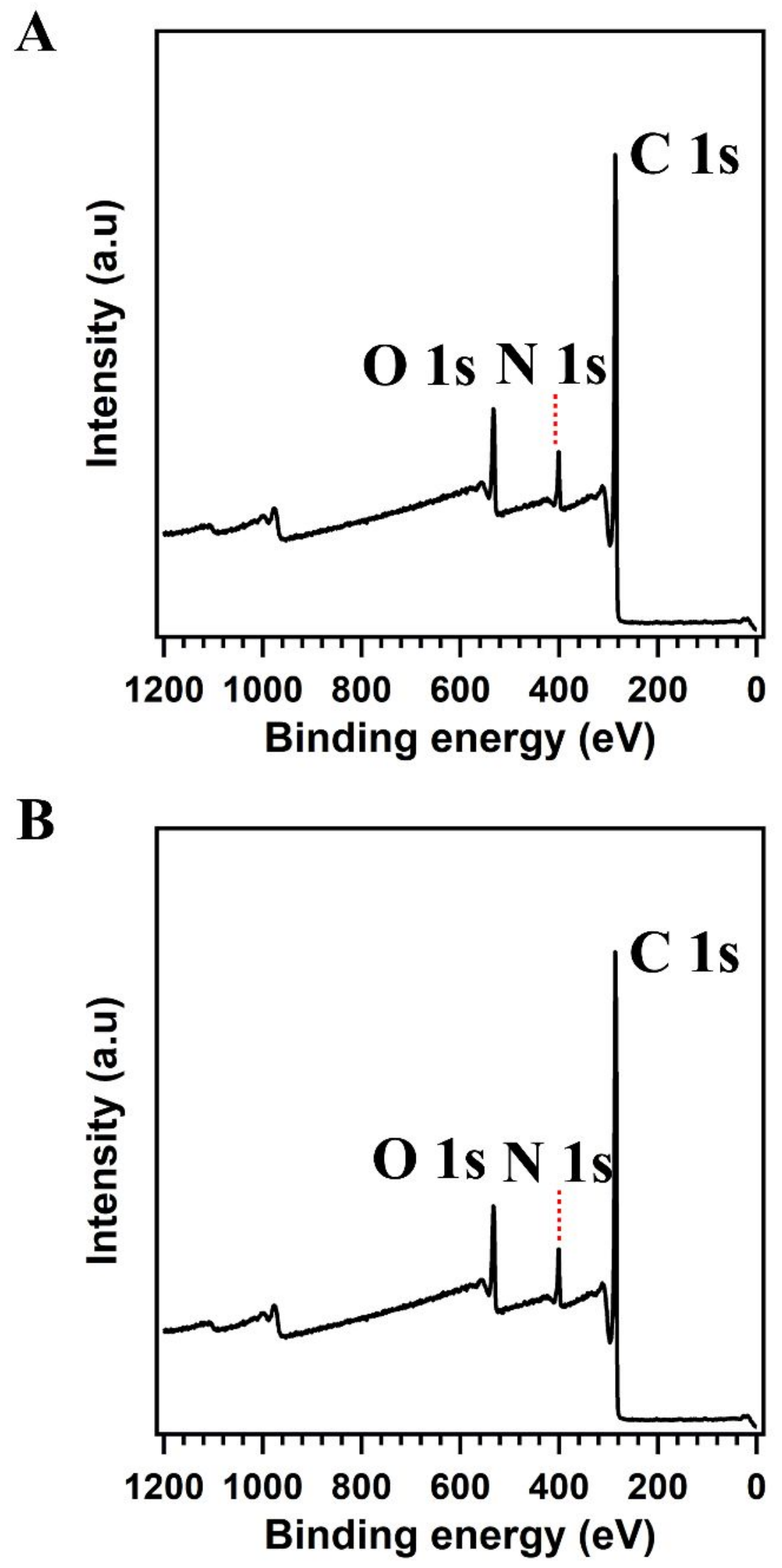

Figure S12. XPS results of TP-BPD-COF (A) and TP-BPY-COF (B). 
A

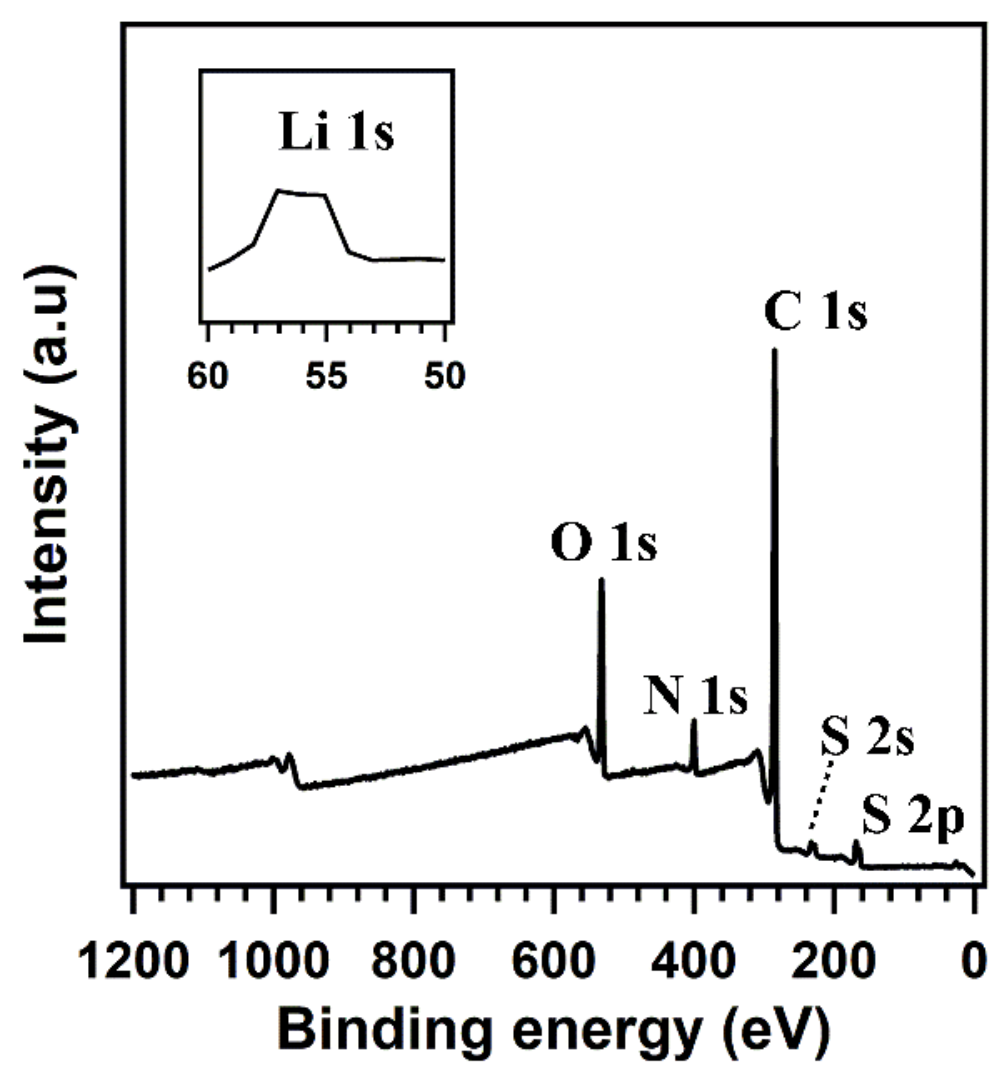

B

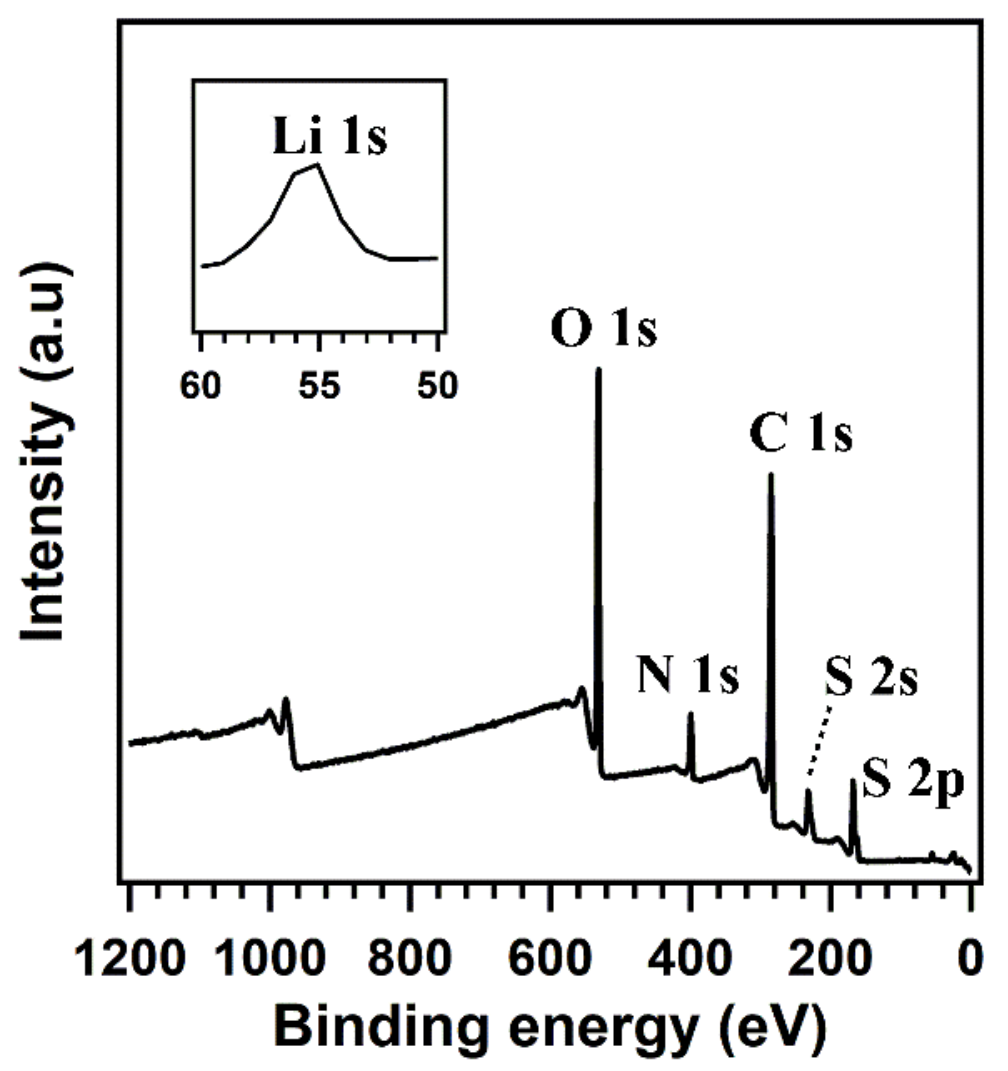

Figure S13. XPS results of $\mathrm{Li}_{2} \mathrm{~S}_{6} @ \mathrm{TP}-\mathrm{BPD}-\mathrm{COF}(\mathrm{A})$ and $\mathrm{Li}_{2} \mathrm{~S}_{6} @ \mathrm{TP}-\mathrm{BPY}-\mathrm{COF}(\mathrm{B})$. 


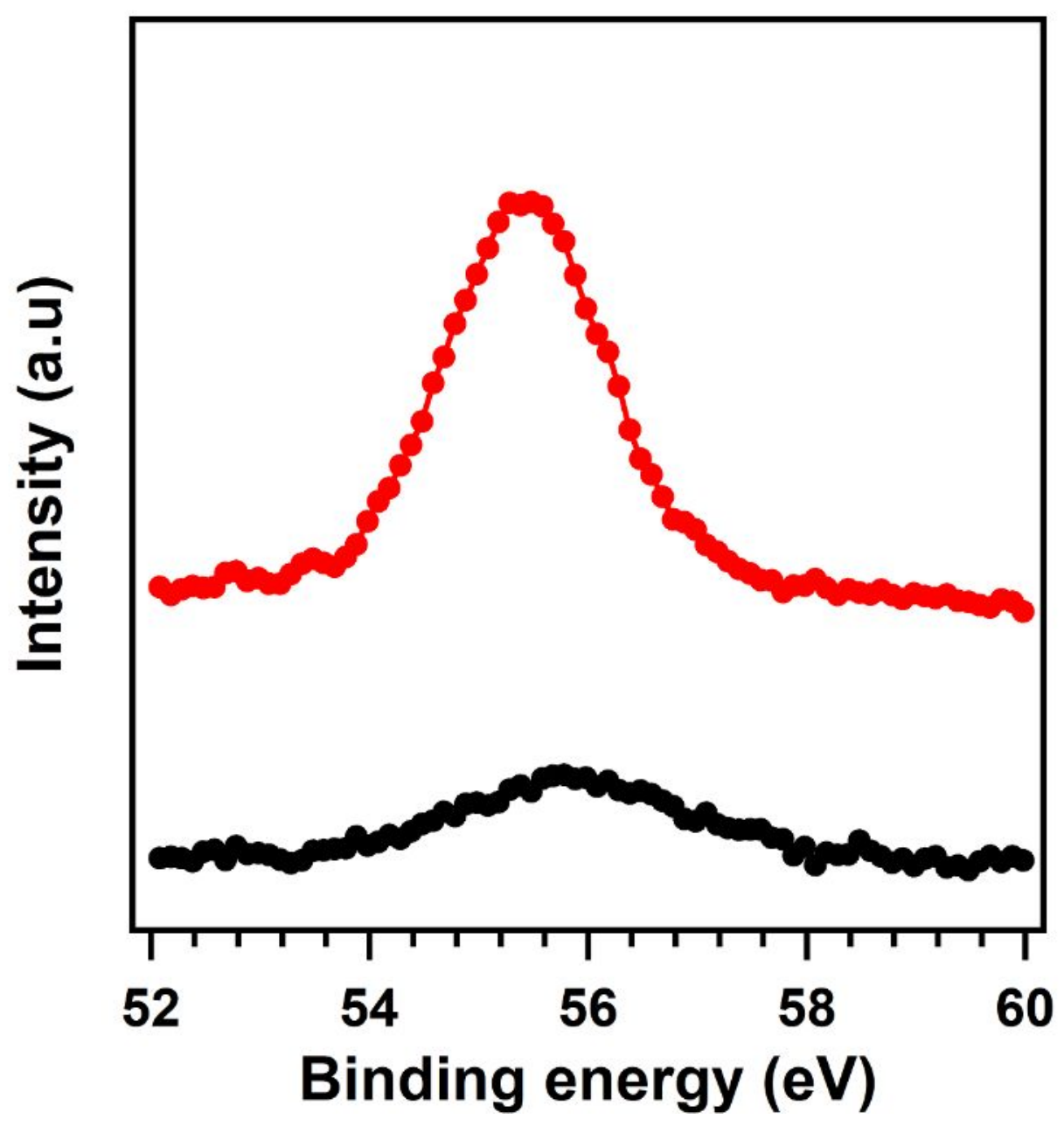

Figure S14. XPS spectra in Li regions of $\mathrm{Li}_{2} \mathrm{~S}_{6} @ \mathrm{TP}-\mathrm{BPD}-\mathrm{COF}$ (black) and $\mathrm{Li}_{2} \mathrm{~S}_{6} @ \mathrm{TP}-\mathrm{BPY}-$ COF (red). 

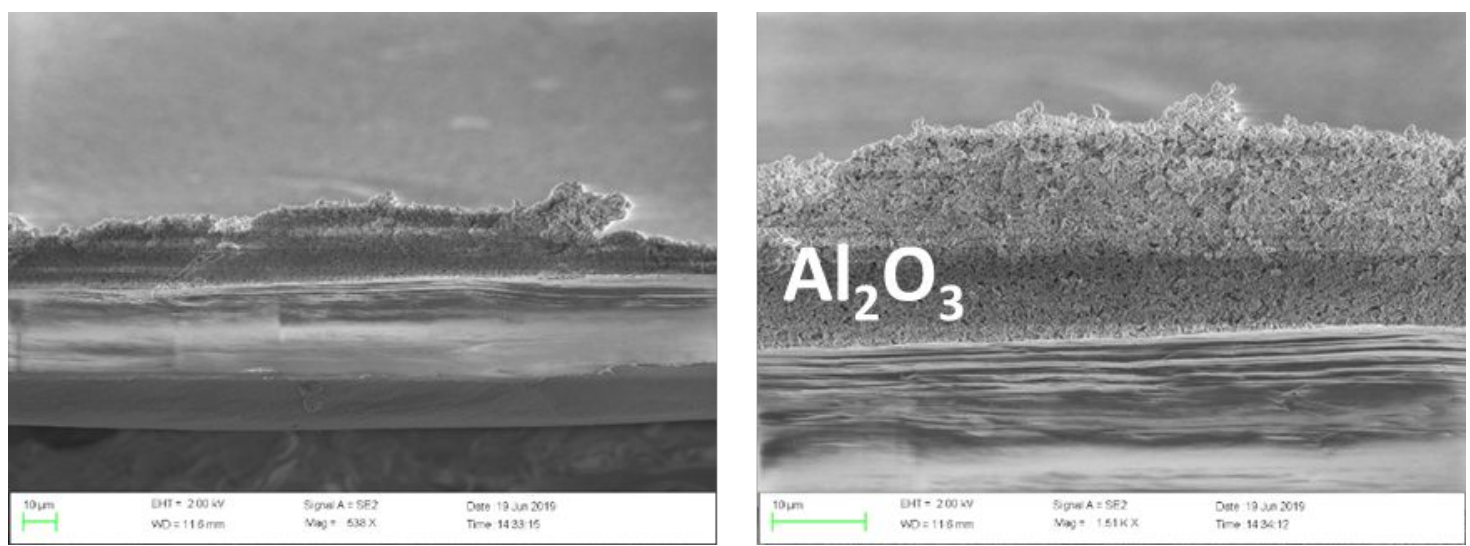

Figure S15. The SEM images of pure separator. 


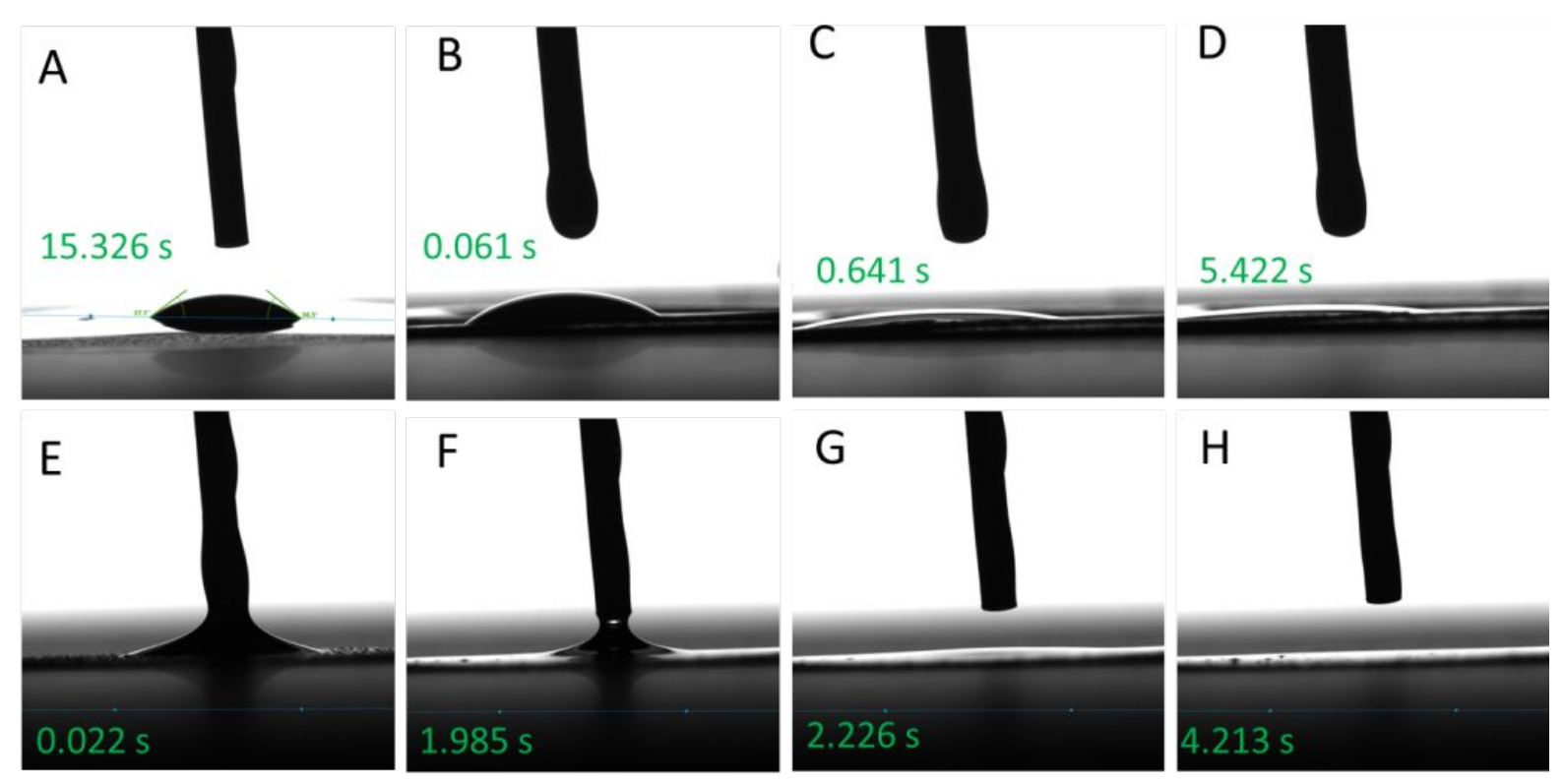

Figure S16. Contact angle measurements (German kruss, DSA30E): The experiments were conducted with electrolyte ( $1 \mathrm{M}$ LITFSI, DOL:DME=1:1, $\left.1 \mathrm{wt} \% \mathrm{LiNO}_{3}\right)$ in the air at room temperature. A) Celgard 2400 without coating $\mathrm{Al}_{2} \mathrm{O}_{3}$; B), C) and D) are Celgard 2400 with $\mathrm{Al}_{2} \mathrm{O}_{3}$ coating at different moment; E), F), G) and H) are TP-BPY-COF@LSBs separator at different moment. 

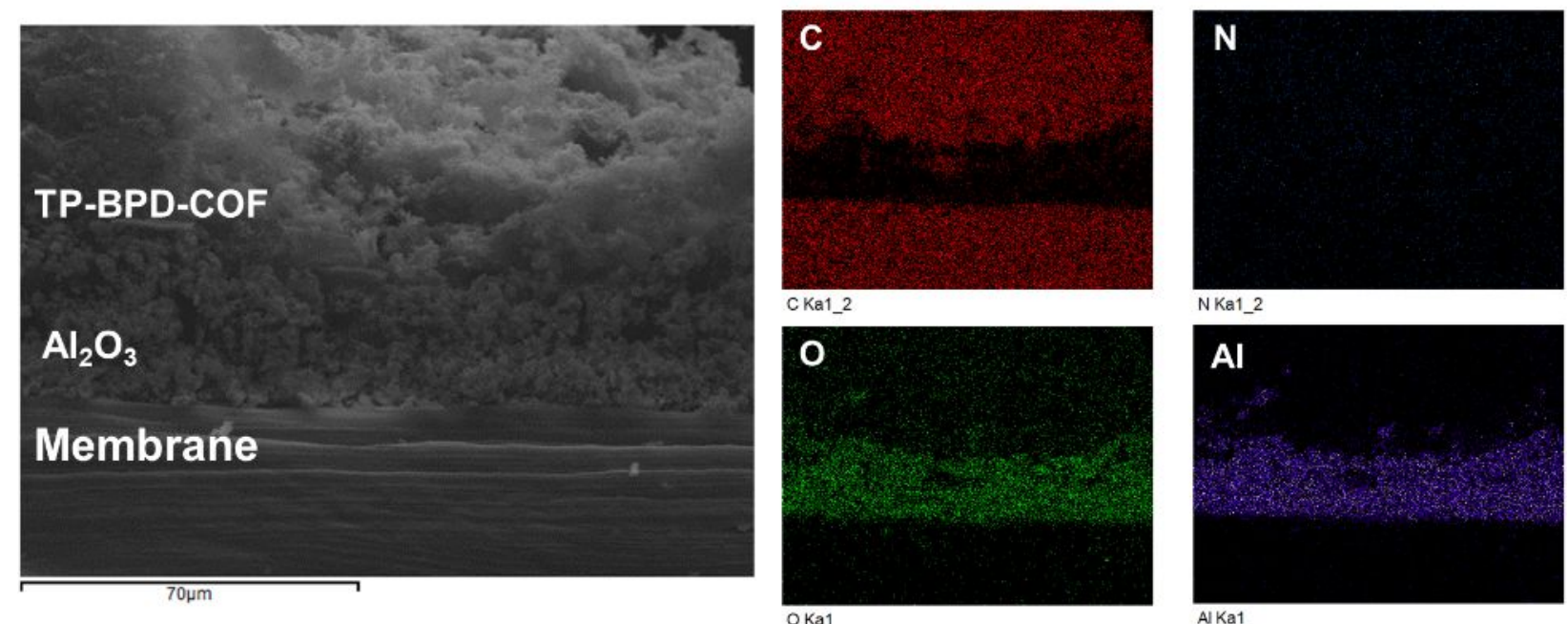

Figure S17. SEM-mapping images of TP-BPD-COF based separator. 


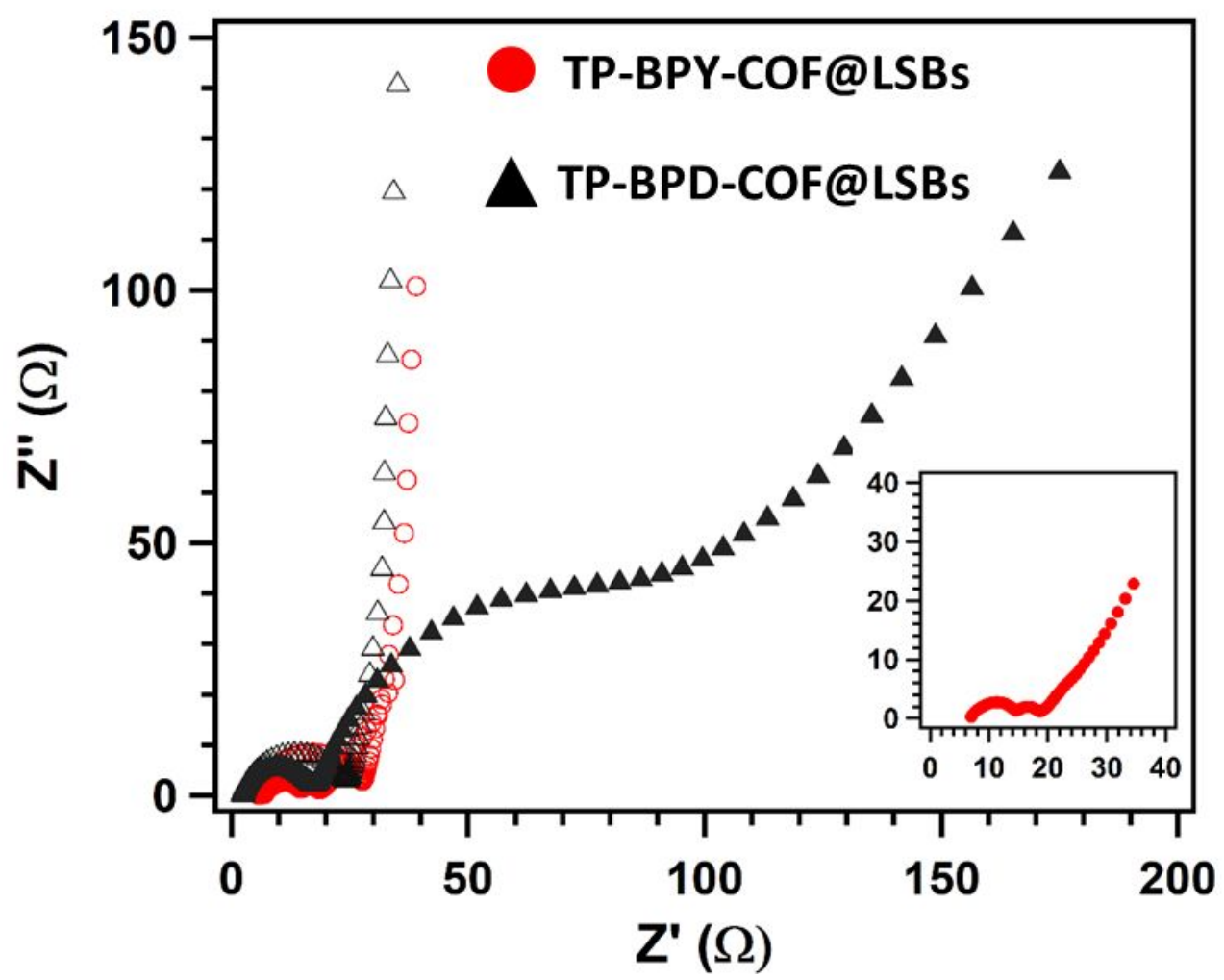

Figure S18. Nyquist plots of the EIS spectra of TP-BPY-COF@LSBs and TP-BPD-COF@LSBs the before cycling (open symbols) and after 100 cycles (solid symbols) at $0.2 \mathrm{C}$. 


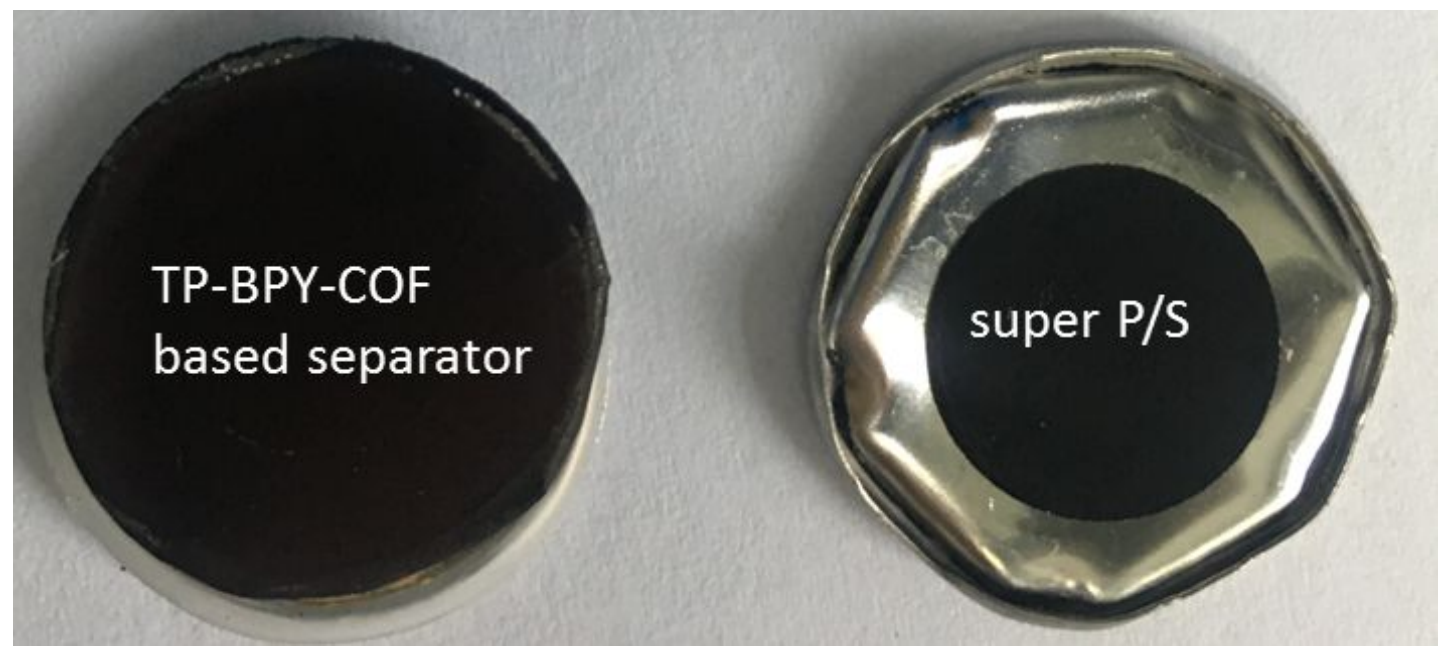

Figure S19. Digital photos of modified separator after 250 cycles at $1 \mathrm{C}$. 

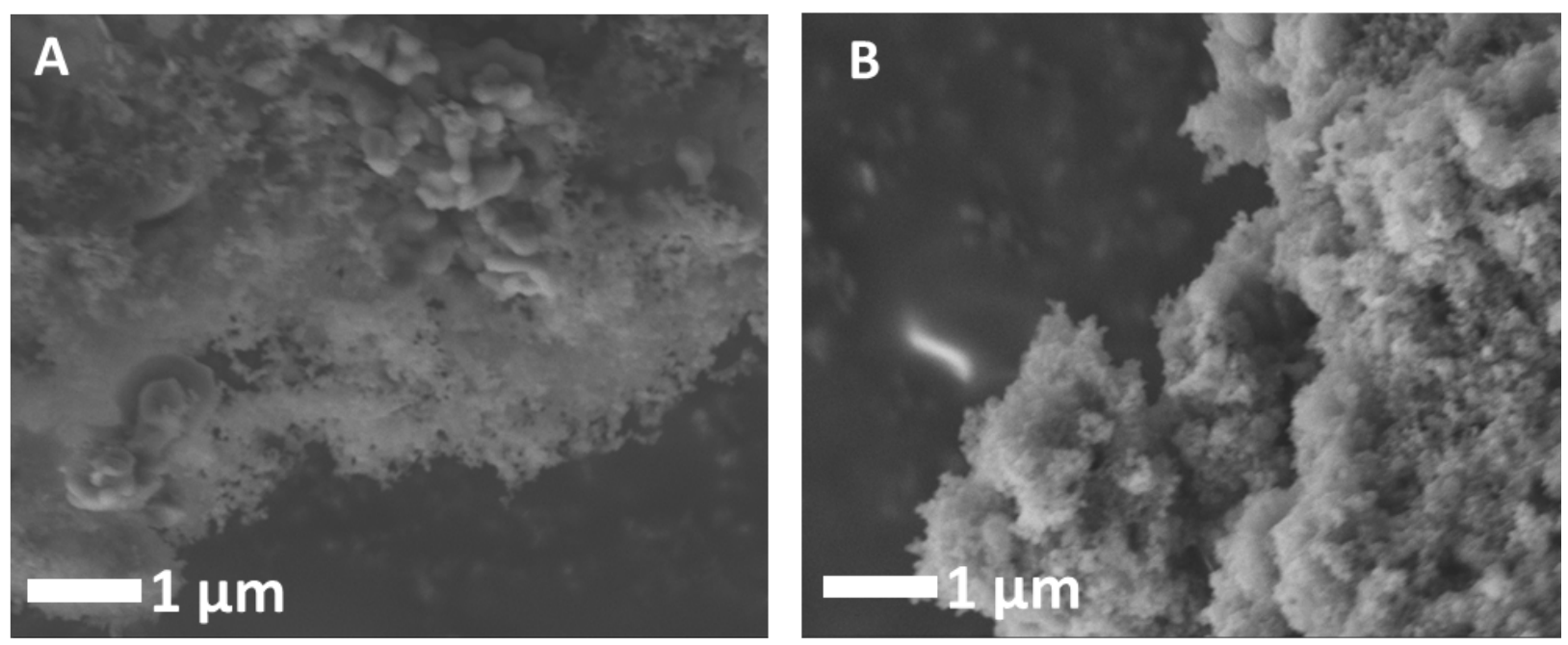

Figure S20. SEM images of TP-BPY-COF before (A) and after (B) 250 cycles at $1 \mathrm{C}$. 


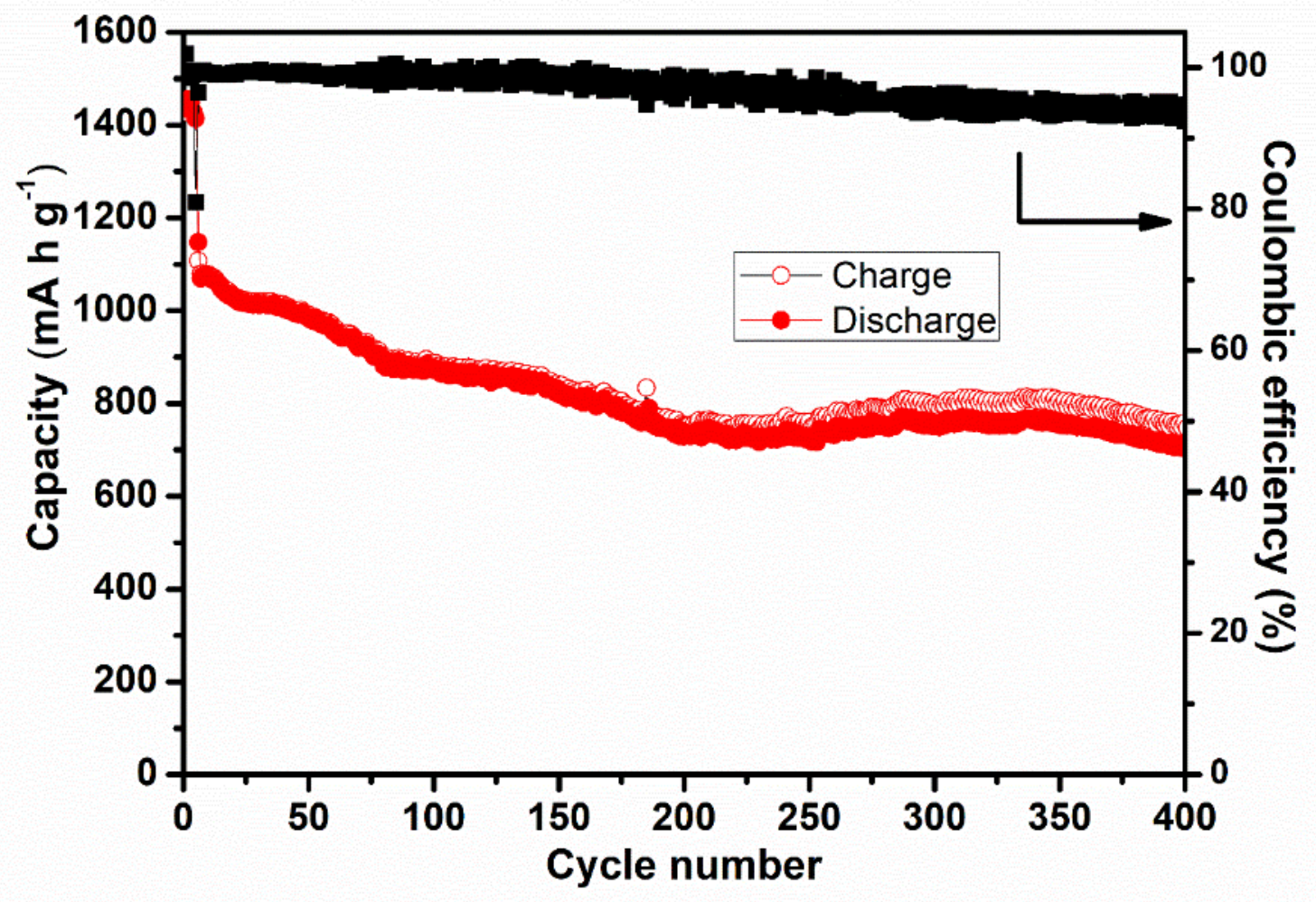

Figure S21. Cycle performance of TP-BPY-COF@LSBs at 2 C. 


\section{Section 4. Supporting Tables}

Table S1. COF-based lithium-sulfur batteries performance.

\begin{tabular}{|c|c|c|c|}
\hline Batteries & Cycle Performance & Rate performance & Reference \\
\hline $\begin{array}{c}\text { TP-BPY- } \\
\text { COF@LSBs }\end{array}$ & $\begin{array}{c}977 \mathrm{mAh} \mathrm{g}^{-1}(0.2 \mathrm{C}, 100 \text { cycles }) \\
\left.826 \mathrm{mAh} \mathrm{g}^{-1} \text { (1 C, } 250 \text { cycles }\right)\end{array}$ & $\begin{array}{c}1026 \mathrm{mAh} \mathrm{g}^{-1}(0.5 \\
\mathrm{C}) \\
572 \mathrm{mAh} \mathrm{g}^{-1}(5 \mathrm{C})\end{array}$ & This work \\
\hline S-COF-V & $\begin{array}{c}959 \mathrm{mAh} \mathrm{g}^{-1} \\
(0.2 \mathrm{C}, 100 \text { cycles })\end{array}$ & $\begin{array}{c}1045 \mathrm{mAh} \mathrm{g}^{-1}(0.5 \\
\mathrm{C}) \\
431 \mathrm{mAh} \mathrm{g}^{-1}(5 \mathrm{C})\end{array}$ & S5 \\
\hline COF-rGo & $\begin{array}{c}\approx 600 \mathrm{mAh} \mathrm{g}^{-1} \\
(1 \mathrm{C}, 250 \text { cycles })\end{array}$ & $<400 \mathrm{mAh} \mathrm{g}^{-1}(3 \mathrm{C})$ & S6 \\
\hline Por-COF/S & $\begin{array}{c}\approx 633 \mathrm{mAh} \mathrm{g}^{-1} \\
(0.5 \mathrm{C}, 200 \text { cycles })\end{array}$ & $730 \mathrm{mAh} \mathrm{g}^{-1}(0.5 \mathrm{C})$ & S7 \\
\hline $\begin{array}{l}\text { DMTA- } \\
\text { COF }\end{array}$ & $\begin{array}{c}797 \mathrm{mAh} \mathrm{g}^{-1} \\
(0.5 \mathrm{C}, 100 \text { cycles })\end{array}$ & $\begin{array}{c}925 \mathrm{mAh} \mathrm{g}^{-1}(0.5 \mathrm{C}) \\
455 \mathrm{mAh} \mathrm{g}^{-1}(5 \mathrm{C})\end{array}$ & S8 \\
\hline $\begin{array}{l}\text { COF-1 NN } \\
\text { interlayer }\end{array}$ & $\begin{array}{c}<900 \mathrm{mAh} \mathrm{g}^{-1} \\
(0.2 \mathrm{C}, 100 \text { cycles })\end{array}$ & / & S9 \\
\hline S/Azo-COF & $\begin{array}{c}602 \mathrm{mAh} \mathrm{g} \mathrm{g}^{-1} \\
(1 \mathrm{C}, 100 \text { cycles })\end{array}$ & $\begin{array}{c}976 \mathrm{mAh} \mathrm{g}^{-1}(0.5 \mathrm{C}) \\
874 \mathrm{mAh} \mathrm{g}^{-1}(1 \mathrm{C})\end{array}$ & S10 \\
\hline COF-1/S & $\begin{array}{l}929 \mathrm{mAh} \mathrm{g}^{-1}(0.2 \mathrm{C}, 100 \text { cycles }) \\
\left.770 \mathrm{mAh} \mathrm{g}^{-1} \text { (0.5 C, } 200 \text { cycles }\right)\end{array}$ & $\begin{array}{c}822 \mathrm{mAh} \mathrm{g}^{-1}(0.5 \mathrm{C}) \\
620 \mathrm{mAh} \mathrm{g}^{-1}(1 \mathrm{C})\end{array}$ & S11 \\
\hline
\end{tabular}


Table S2. Comparison of the electrochemical performance of state-of-the-art Li-S batteries.

\begin{tabular}{|c|c|c|c|}
\hline Batteries & Cycle Performance & Rate performance & Reference \\
\hline $\begin{array}{c}\text { TP-BPY- } \\
\text { COF@LSBs }\end{array}$ & $\begin{array}{c}977 \mathrm{mAh} \mathrm{g}^{-1} \text { (0.2 C, } 100 \text { cycles) } \\
\left.826 \mathrm{mAh} \mathrm{g}^{-1} \text { (1 C, } 250 \text { cycles }\right)\end{array}$ & $\begin{array}{c}1026 \mathrm{mAh} \mathrm{g}^{-1}(0.5 \mathrm{C}) \\
572 \mathrm{mAh} \mathrm{g}^{-1}(5 \mathrm{C})\end{array}$ & This work \\
\hline S-AQ-G & $\begin{array}{c}938 \mathrm{mAh} \mathrm{g}^{-1} \\
(0.1 \mathrm{C}, 100 \text { cycles })\end{array}$ & l & $\mathrm{S} 12$ \\
\hline PCNF/S/BPQD & $\begin{array}{c}1073 \mathrm{mAh} \mathrm{g}^{-1} \\
(0.1 \mathrm{C}, 200 \text { cycles })\end{array}$ & $\begin{array}{c}1030 \mathrm{mAh} \mathrm{g}^{-1}(0.5 \mathrm{C}) \\
910 \mathrm{mAh} \mathrm{g}^{-1}(1 \mathrm{C})\end{array}$ & S13 \\
\hline $\begin{array}{c}\text { Phosphorene } \\
\text { separator }\end{array}$ & $\begin{array}{c}\approx 800 \mathrm{mAh} \mathrm{g}^{-1} \\
(0.24 \mathrm{C}, 100 \text { cycles })\end{array}$ & $623 \mathrm{mAh} \mathrm{g}^{-1}(2 \mathrm{C})$ & S14 \\
\hline $\begin{array}{l}\mathrm{WS}_{2} / \mathrm{Li}_{2} \mathrm{~S}_{6} \\
\text { catholyte }\end{array}$ & $\begin{array}{c}652 \mathrm{mAh} \mathrm{g}^{-1} \\
(0.2 \mathrm{C}, 100 \text { cycles })\end{array}$ & $380 \mathrm{mAh} \mathrm{g}^{-1}(1 \mathrm{C})$ & S15 \\
\hline NG/S & $\begin{array}{c}848 \mathrm{mAh} \mathrm{g}^{-1} \\
(0.2 \mathrm{C}, 200 \text { cycles })\end{array}$ & $\begin{array}{c}970 \mathrm{mAh} \mathrm{g}^{-1}(0.5 \mathrm{C}) \\
850 \mathrm{mAh} \mathrm{g}^{-1}(2 \mathrm{C})\end{array}$ & S16 \\
\hline $\begin{array}{l}\text { CoP-containing } \\
\text { electrode }\end{array}$ & $\begin{array}{c}835 \mathrm{mAh} \mathrm{g}^{-1} \\
(1 \mathrm{C}, 200 \text { cycles })\end{array}$ & l & S17 \\
\hline $\begin{array}{l}\text { CNF-GA } \\
\text { interlayers }\end{array}$ & $\begin{array}{c}827 \mathrm{mAh} \mathrm{g}^{-1} \\
(1 \mathrm{C}, 250 \text { cycles })\end{array}$ & $544 \mathrm{mAh} \mathrm{g}^{-1}(3 \mathrm{C})$ & S18 \\
\hline $\mathbf{S @ N i / F e ~ L D H ~}$ & $\begin{array}{c}724 \mathrm{mAh} \mathrm{g}^{-1} \\
(0.2 \mathrm{C}, 200 \text { cycles })\end{array}$ & $\begin{array}{c}853 \mathrm{mAh} \mathrm{g}^{-1}(0.5 \mathrm{C}) \\
633 \mathrm{mAh} \mathrm{g}^{-1}(2 \mathrm{C})\end{array}$ & S19 \\
\hline $\begin{array}{c}\text { G@CB-coated } \\
\text { separator }\end{array}$ & $\begin{array}{c}678 \mathrm{mAh} \mathrm{g}^{-1} \\
(0.5 \mathrm{C}, 300 \text { cycles })\end{array}$ & $\begin{array}{c}898 \mathrm{mAh} \mathrm{g}^{-1}(0.5 \mathrm{C}) \\
799 \mathrm{mAh} \mathrm{g}^{-1}(2 \mathrm{C})\end{array}$ & S20 \\
\hline S@Co/N-PCNSs & $\begin{array}{c}633 \mathrm{mAh} \mathrm{g}^{-1} \\
(0.5 \mathrm{C}, 300 \text { cycles })\end{array}$ & $\begin{array}{c}871 \mathrm{mAh} \mathrm{g}^{-1}(0.5 \mathrm{C}) \\
520 \mathrm{mAh} \mathrm{g}^{-1}(5 \mathrm{C})\end{array}$ & S21 \\
\hline $\begin{array}{l}\text { N,S-codoped } \\
\text { graphene } \\
\text { electrode }\end{array}$ & $\begin{array}{c}670 \mathrm{mAh} \mathrm{g}^{-1} \\
(0.5 \mathrm{C}, 200 \text { cycles })\end{array}$ & $\begin{array}{c}912 \mathrm{mAh} \mathrm{g}^{-1}(0.5 \mathrm{C}) \\
430 \mathrm{mAh} \mathrm{g}^{-1}(2 \mathrm{C})\end{array}$ & S22 \\
\hline
\end{tabular}




\section{Section 5. Supporting References}

S1. Aiyappa, H. B.; Thote, J.; Shinde, D. B.; Banerjee, R.; Kurungot, S. Cobalt-Modified Covalent Organic Framework as a Robust Water Oxidation Electrocatalyst Chem. Mater. 2016, 28, 4375-4379.

S2. Shinde, D. B.; Aiyappa, H. B.; Bhadra, M.; Biswal, B. P.; Wadge, P.; Kandambeth, S.; Garai, B.; Kundu, T.; Kurungot, S.; Banerjee, R. A mechanochemically synthesized covalent organic framework as a proton-conducting solid electrolyte J. Mater. Chem. A, 2016, 4, 26822690.

S3. S.R. Das, S.B. Majumder, R.S. Katiyar, Kinetic analysis of the Li+ ion intercalation behavior of solution derived nano-crystalline lithium manganate thin films J. Power Sources, 2005, $139,261-268$.

S4. Zhang, K.; Wang, L.; Cai, W.; Chen, L.F.; Wang, D.; Chen, Y.; Pan, H.; Wang, L.; Qian, Y. Pyridinic-and Pyrrolic-Nitrogen enriched Carbon as Polysulfide Blocker for HighPerformance Lithium-Sulfur Batteries Inorg. Chem. Front., 2019, 6, 955-960.

S5. Jiang, Q.; Li, Y.; Zhao, X.; Xiong, P.; Yu, X.; Xu, Y.; Chen, L. Inverse-vulcanization of vinyl functionalized covalent organic frameworks as efficient cathode materials for $\mathrm{Li}-\mathrm{S}$ batteries. J. Mater. Chem. A, 2018, 6, 17977.

S6. Jiang, C.; Tang, M.; Zhu, S.; Zhang, J.; Wu, Y.; Chen, Y.; Xia, C.; Wang, C.; Hu, W. Constructing Universal Ionic Sieves via Alignment of Two-Dimensional Covalent Organic Frameworks (COFs). Angew. Chem. Int. Ed. 2018, 57, 16072-16076.

S7. Liao, H.; Wang, H.; Ding, H.; Meng, X.; Xu, H.; Wang, B.; Ai, X.; Wang, C. A 2D porous porphyrin-based covalent organic framework for sulfur storage in lithium-sulfur batteries. $J$. Mater. Chem. A, 2016, 4, 7416-7421.

S8. Wang, J.; Si, L.; Wei, Q.; Hong, X.; Cai, S.; Cai, Y. Covalent Organic Frameworks as the Coating Layer of Ceramic Separator for High-Efficiency Lithium-Sulfur Batteries. ACS Appl. Nano Mater. 2018, 1, 132-138.

S9. Yoo, J.; Cho, S.-J.; Jung, G. Y.; Kim, S. H.; Choi, K.-H.; Kim, J.-H.; Lee, C. K.; Kwak, S. K.; Lee, S.-Y. COF-Net on CNT-Net as a Molecularly Designed, Hierarchical Porous Chemical Trap for Polysulfides in Lithium-Sulfur Batteries. Nano Letters 2016, 16, 3292-3300.

S10. Yang, X.; Dong, B.; Zhang, H.; Ge, R.; Gao, Y.; Zhang, H. Sulfur impregnated in a mesoporous covalent organic framework for high performance lithium-sulfur batteries. RSC Advances 2015, 5, 86137-86143.

S11. Ghazi, Z. A.; Zhu, L.; Wang, H.; Naeem, A.; Khattak, A. M.; Liang, B.; Khan, N. A.; Wei, Z.; Li, L.; Tang, Z. Efficient Polysulfide Chemisorption in Covalent Organic Frameworks for High-Performance Lithium-Sulfur Batteries. Adv. Energy Mater. 2016, 6, 1601250. 
S12. Li, G.; Wang, X.; Seo, M. H.; Li, M.; Ma, L.; Yuan, Y.; Wu, T.; Yu, A.; Wang, S.; Lu, J.; Chen, Z. Chemisorption of polysulfides through redox reactions with organic molecules for lithium-sulfur batteries. Nature Communications 2018, 9, 705.

S13. Xu, Z.-L.; Lin, S.; Onofrio, N.; Zhou, L.; Shi, F.; Lu, W.; Kang, K.; Zhang, Q.; Lau, S. P. Exceptional catalytic effects of black phosphorus quantum dots in shuttling-free lithium sulfur batteries. Nature Communications 2018, 9, 4164.

S14. Sun, J.; Sun, Y.; Pasta, M.; Zhou, G.; Li, Y.; Liu, W.; Xiong, F.; Cui, Y. Entrapment of Polysulfides by a Black-Phosphorus-Modified Separator for Lithium-Sulfur Batteries. Adv. Mater. 2016, 28, 9797-9803

S15. Babu, G.; Masurkar, N.; Al Salem, H.; Arava, L. M. R. Transition Metal Dichalcogenide Atomic Layers for Lithium Polysulfides Electrocatalysis. J. Am. Chem. Soc. 2017, 139, 171178.

S16. Hou, T.-Z.; Xu, W.-T.; Chen, X.; Peng, H.-J.; Huang, J.-Q.; Zhang, Q. Lithium Bond Chemistry in Lithium-Sulfur Batteries. Angew. Chem. Int. Ed. 2017, 56, 8178-8182.

S17. Zhong, Y.; Yin, L.; He, P.; Liu, W.; Wu, Z.; Wang, H. Surface Chemistry in Cobalt Phosphide-Stabilized Lithium-Sulfur Batteries. J. Am. Chem. Soc. 2018, 140, 1455-1459.

S18. Tu, S.; Chen, X.; Zhao, X.; Cheng, M.; Xiong, P.; He, Y.; Zhang, Q.; Xu, Y. A PolysulfideImmobilizing Polymer Retards the Shuttling of Polysulfide Intermediates in Lithium-Sulfur Batteries. Adv. Mater. 2018, 30, 1804581.

S19. Zhang, J.; Li, Z.; Chen, Y.; Gao, S.; Lou, X. W. Nickel-Iron Layered Double Hydroxide Hollow Polyhedrons as a Superior Sulfur Host for Lithium-Sulfur Batteries. Angew. Chem. Int. Ed. 2018, 57, 10944-10948.

S20. Xie, J.; Peng, H.-J.; Huang, J.-Q.; Xu, W.-T.; Chen, X.; Zhang, Q. A Supramolecular Capsule for Reversible Polysulfide Storage/Delivery in Lithium-Sulfur Batteries. Angew. Chem. Int. Ed. 2017, 56, 16223-16227.

S21. Liu, S.; Li, J.; Yan, X.; Su, Q.; Lu, Y.; Qiu, J.; Wang, Z.; Lin, X.; Huang, J.; Liu, R.; Zheng, B.; Chen, L.; Fu, R.; Wu, D. Superhierarchical Cobalt-Embedded Nitrogen-Doped Porous Carbon Nanosheets as Two-in-One Hosts for High-Performance Lithium-Sulfur Batteries. Adv. Mater.2018, 30, 1706895.

S22. Zhou, G.; Paek, E.; Hwang, G. S.; Manthiram, A. Long-life Li/polysulphide batteries with high sulphur loading enabled by lightweight three-dimensional nitrogen/sulphur-codoped graphene sponge. Nat. Commun. 2015, 6, 7760. 